\title{
Petro-Geochemical and Statistic Studies of the Nimba Region in the Republic of Guinea
}

\author{
Mohamed Samuel Moriah CONTE ${ }^{1 *}$, Abdellah BOUSHABA ${ }^{2}$ and Ali MOUKADIRI ${ }^{1}$ \\ ${ }^{1}$ Natural Resources Geoscience Laboratory (LGRN) / Dhar El Marhaz Faculty of Sciences / Sidi Mohamed Ben Abdellah University of Fez \\ ${ }^{2}$ Laboratory for Advanced Systems and Technologies Integration (LISTA) / Dhar El Marhaz Faculty of Sciences / Sidi Mohamed Ben Abdellah University \\ of Fez
}

Received 02 Jan 2018, Accepted 03 March 2018, Available online 06 March 2018, Vol.6 (March/April 2018 issue)

\begin{abstract}
Summary: Guinea is described as a geological scandal, due to the richness of its soil, its subsoil, and its complexity. But this geology is poorly studied and under exploited and the region of Nimba hardly escapes to this fact. Located in the south-east of the country, Nimba is about $60 \mathrm{~km}$ long and $25 \mathrm{~km}$ wide, it belongs to the Precambrian domain (Archean basement (Craton) and Proterozoic) and is characterized by volcano-sedimentary rocks. In addition to mineral diversity, shear, brecciation and fault structures are observed in the rocks (Nion District), while they are not observable in rocks of other parts of the region. Large deposits of iron (magnetite, hematite, goethite) occupy the summits of the region. Granites exhibit magmatic differentiation whereas amphibolites occupy the MORB domain set up by rifting. The major elements exhibit perfect positive and negative linear relationships, while the rare earths exhibit perfect positive linear relationships. The PCA of the major elements confirms the differentiation of granitic rocks while the amphibolites do not show differentiation due to weathering.
\end{abstract}

Objective: Geological cross sections of the Nimba range, petrographic studies of the different rocks and mineralogical description of these rocks were realized. And this to know the context of setting up of the region using the results of chemical analysis, the knowing of the different correlations which allow to visualize a space with $p$ dimensions using spaces of smaller dimensions of the Geochemical data.

Materials and Methods: This study in the Nimba region was done in two stages. The first stage performed in the field (period from November 25 to December 20, 2015 and from January 23 to February 15, 2017); it permits to collect the samples, the realization of two geological cross sections (one in the South near the border between the Republic of Guinea and Liberia, and one in the North in the fan zone of the region). The second step, carried out in the laboratories, with the digitalization of the two (2) geological cross sections (using Canvas version: 11Build 1252), then a microscopic description of the samples and the analysis of the different spectroscopies and diffractograms of the rocks of the Nimba region (using the Bruker Raman SENTERRA type spectroscope and the Pert Pro Panalytical X-type diffractometer). The rocks were analyzed geochemically by ICP-AES and ICP-MS. Finally, the geochemical data is processed by statistical methods (using Statgraphic Centurion software version_16.1.11).

Results: The Nimba region contains two (2) groups of rocks, the first group clear and SiO2 rich (Gneiss, Granites, and Quartzites) and the second group is dark and SiO2 poor (Muscovitites, Amphibolites). But il also contains Itabirite-type iron deposits (Banded Irons Formations: BIFs) which occupy the main peaks of the Nimba region chain. These rocks contain minerals that are abundant in some and rare in others or even absent (Table 1). Next, they reveal that the quartzite facies are characterized by open muscovite crystals in the form of " $S$ " slits and crystals of quartz, $K$ feldspar and " $\mathrm{C}$ " plagioclases that give the " $\mathrm{C} / \mathrm{S}$ " structures and hence the formation of "Shears Zones" (District of Nion, photo 2r). Amphibolitic rocks (Nion District) reveal both a breccia zone and a fault zone (Figure $2 w$ and $2 x$ ). The breccia zone constitutes the phase S1 which is filled by the grunerite and the fault zone constitutes the phase S2 filled with microcrystals of quartz, magnetite, feldspars and grunerite. This phase S2 is characterized by the displacement of the blocks due to the fault activity. On the other hand, in the district of Gbie, the amphibolites do not contain zones of breccias or of faults, but they reveal the arrangement of the minerals (figure $2 z$ and $2 a a$ ). The spectroscopies and diffractograms of the different rocks of the Nimba region confirm the results obtained from the petrographic description but also the appearance of new minerals (Table 2). The chemical rocks analyses of the Nimba region reveal that they are sub-alkaline. The amphibolites are tholeiitics, occupying the area of MORBs, with REE spectra (normalized to chondrites of N-MORBs derived from depleted upper mantle) showing depletion in LREE and almost flat in HREE. The granitics rocks are calco-alkaline, peraluminous, marked by the decrease of minerals ferromagnesian, ferro-titanic oxides and plagioclase with an increase of the alkali feldspar content. The spectra of REE of granitic rocks chondrite normalized at 
the chondrite show both negative in Eu anomalies suggesting on one hand that the plagioclase was fractioned and positive anomalies in EU that indicates on the other hand that the plagioclase has not been fractioned. All these spectra present the enrichment in LREE and a poverty in HREE. The Bravais-Pearson correlation of the major elements of amphibolitic and granitic rocks shows that Al2O3 does not correlate with other major elements and P2O5 correlates only with TiO2. The rest of the major elements have both positive linear relations and perfect negatives with $P$-Values less than 0.05. The Pearson-Pearson correlation of REE of amphibolitic and granitic rocks shows perfect positive linear relationships with P-Values less than 0.05. The Principal Components Analysis (PCA) of the major elements of amphiboles and granites has three groups of major elements, the first formed of SiO2, Na2O and K2O which correlate with each other to the component 1 . The second formed of $\mathrm{MgO}, \mathrm{TiO} 2, \mathrm{MnO}, \mathrm{Fe} 2 \mathrm{O} 3 \mathrm{t}$ and $\mathrm{CaO}$ correlate with each other at component 1; these groups are anti-correlated with respect to component 2. The third group of major elements consists of Al2O3 and P2O5 which correlate neither with component 2 nor with the first two groups of major elements. Only TiO2 correlates with P2O5. Finally, the amphibolites have an affinity with the second group and do not exhibit magmatic differentiation due to weathering and weather conditions. The granitic rocks have an affinity with the first group, they are distributed according to the magmatic differentiation. The Principal Component Analysis (PCA) of REE of amphibolitic and granitic rocks has two groups: the first formed of $\mathrm{Ce}, \mathrm{La}, \mathrm{Pr}, \mathrm{Nd}, \mathrm{Sm}$, Gd, and Eu which correlate with each other at component 1. The second, Tb, Dy, Er, Ho, Tm, Yb and Lu that correlate with each other at component 1. These two groups of chemical elements are not anti-correlated to component 2 , which is confirmed by the hypothesis of the linear correlation method.

Conclusions: The region of Nimba belongs to the precambrian domain, it is largely correlated with those of Brazil and Venezuella. It is formed by metavolcanic and metasedimentary rocks: quartzitic rocks characterized by ' $C / S$ ' shear and zoned zircon; grunerite amphibolites (cummingtonite family) rich in iron and associated with large iron deposits in fault and breccia zones. These amphibolites are located in the MORB field, which was emplaced by rifting, while the granitic rocks were emplaced by magmatic differentiation. This region is rich in iron deposits that occupy the main peaks and whose establishment was made by precipitation of a silico-ferruginous gel in a closed basin, virtually protected from other terrigenous inputs. This precipitation of dissolved iron results from an enrichment in atmospheric 02 characteristic of the Archean and Paleoproterozoic.

Keywords: Mineralogy, Petrography, Geochemistry, PCA, Shear-zones, Itabirite iron deposits, MORB, Precambrian, Nimba region, Guinea.

\section{Introduction}

Guinea is located in the complex junction area of large geological structures of different ages. Its geology is largely correlated with that south of West African Craton 'WAC'. It can be summarized in two great sets: a Precambrian basement, also called the Eburnean Shield [1] or the Kenema-Man Craton [2], which includes the Archean, Early Proterozoic and Late Riphean - Vendian phases; and a Paleozoic sedimentary cover traversed by basic and ultrabasic intrusions emplaced in the Mesozoic, covered in some places by Cenozoic deposits [Figure 1].

In Guinea, the region of the Nimba range belongs to the Leóno-Liberian Massif, whose orthogneiss age is comparable to that of the Reguibat uprising orthogneiss in Mauritania [3]. Guinea is truly a geological scandal. For many geologists, it remains a curiosity to discover and an opportunity to seize for mining companies. However, the Guinean subsoil is not at the end of its surprises. Its geology is probably known only on a large scale. Research and geological studies are still ongoing. Poorly known regions are in the planning stages or in the idea of an

*Corresponding author's ORCID ID: 0000-0002-5584-0205 DOI: https://doi.org/10.14741/ijmcr/v.6.2.2 exploration project, and some of them still look very promising. Despite a good level of outcropping in the Nimba region, it is not satisfactorily studied [4]. For the first time, this region was studied by [5] who identified a bedrock of ferruginous quartzites and various greenschists, called "Nimba series". Then, the region was studied by [6], [7], [8], [9], [10] who performed a geological survey on the N'Zérékoré sheet map, after which they differentiated two rock complexes composing the mountainous massif of Nimba:

- a meta-sedimentary complex;

- a meta-volcanic complex or basic stratified intrusions.

The present work falls within this framework, to concisely study the petro-geochemistry, mineralogy and rock statistics of the Nimba range region, which is an enigma for geologists.

During our fieldwork in the Nimba region [from November 25 to December 20, 2015 and from January 23 to February 15, 2017], two geological cross sections were made, samples were collected and analyzed petrographically, mineralogically and geochemically. Then a statistical treatment [Pearson-Bravais correlation and 
principal components analysis] was carried out on these geochemical data.

The two (2) geological cross sections carried out in the field were digitized using the Canvas drawing software, version 11Bluid 1252 . Then, the thin section studies were carried out in the Department of Geology of the Faculty of Sciences Dhar El Mahraz of Sidi Mohamed Ben Abdellah University of Fez [Morocco]. Approximately sixty [60] thin sections were examined under a polarizing microscope, which allowed the selection of twenty [20] samples for Raman spectroscopy and X-ray diffraction analyzes. These analyzes were performed at the Laboratoire of the "Cité d 'Innovation [CURI]", University of Sidi Mohamed Ben Abdellah of Fez [Morocco]. Raman analyzes were performed using the Bruker Raman SENTERRA spectroscope with a spectral region of 90 to $3200 \mathrm{~cm}^{-1}$ and excitation at $785 \mathrm{~nm}$, a spectral resolution of $3 \mathrm{~cm}^{-1}$, a laser of $785 \mathrm{~nm}$ and $100 \mathrm{~mW}$. For all spectra, the band position and bandwidth assignments were determined using Trinocular software. The spectra were obtained by submitting thin sections or macroscopic samples to an average of five point analyzes at the rate of 10s each. With an X-ray Pert Pro Panalytical diffractometer, during the analysis, the samples are bombarded by an X-ray beam of known wavelength, produced here by a copper anticathode $(\lambda K \alpha 1=1.5405$ Angstrom, $\lambda K \alpha 2=1.5443$ Angstrom) powered by a high voltage generator $(45 \mathrm{kV}, 40 \mathrm{~mA})$. The emitted radiation is defined by a system of slits (Soller, divergence and antidiffusion) and windows located before and after the sample. A detector measures the intensity of diffracted $X$ ray radiation in certain directions under Bragg conditions. The recording made (called diffractogram) is the curve of the intensity of X-rays diffracted as a function of the diffraction angles. Twenty (20) samples (amphibolites and granites) were analyzed at SARAM CNRS of Nancy: by Atomic Emission Spectrometry Coupled to an ICP-AES induced plasma for major elements and by ICP-induced plasma Coupled Mass Spectrometry ICP-MS for trace elements.

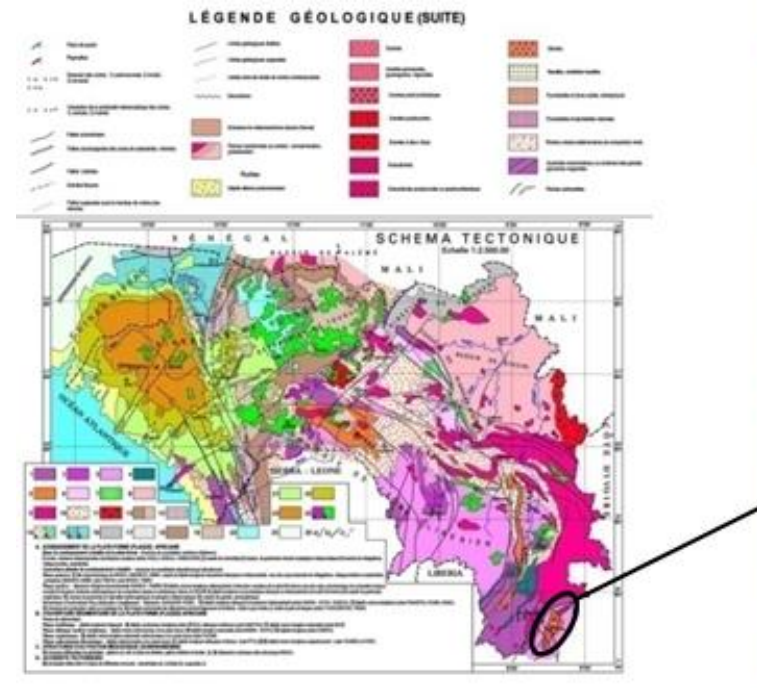

Figure 1: Geological map of Guinea [5]

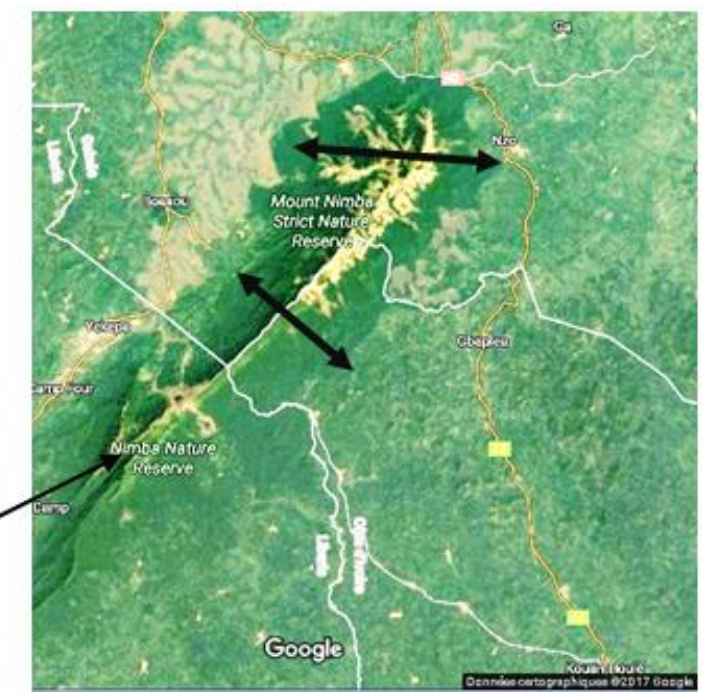

Photo 1: Satellite image of the Nimba region [11]
Depending on the amounts, the uncertainty on the assay is between less than $1 \%$ to $10 \%$ for the major elements and less than $5 \%$ to $20 \%$ for the trace elements. The method involves ionizing the sample by injecting it into an argon plasma. The substances to be analyzed are transformed into ions by a kind of extremely hot flame: up to $8000 \mathrm{~K}$, but generally around $6000 \mathrm{~K}$. The samples are introduced into the plasma in a condensed form (solid) and will therefore undergo a change of state from solid to liquid (melting). The introduction takes place in the center of the plasma, parallel to the flow of plasma gas. The sample must be introduced into the plasma in a finely divided form, since the incident powers used (generally less than 2,000 watts) do not make it possible to treat particles larger than one micrometer during their residence time within the plasma. If one wants to analyze a solid, it must first be transformed into a suspension of fine particles, carried by a stream of plasma gas. High frequency (HF) plasma torches are coupled to generators that can provide more than $200 \mathrm{~kW}$. Finally, these geochemical data are processed by statistical methods using the software 'Statgraphic Centurion, version_16.1.11'. The simple linear correlation coefficient, known as Bravais-Pearson, is a method of normalizing the covariance by the product of the standard deviations of the variables.

The correlation coefficient is independent of the units of measure of the variables, which allows the comparisons. The measurement is normalized, it is defined between $-1 \leq r \leq 1$. When $r=1$, the connection between the parameters is linear, positive and perfect, ie the knowledge of $X$ gives us the value of $Y$ (and vice versa); if $r=-1$, the bond is linear and negative. These correlation coefficients measure the force of linear relationship between the variables. Principal Components Analysis (PCA) is a method of the family of data analysis and more generally of multivariate statistics consisting of graphically representing as much information as possible in a table. It allows to visualize a space with $\mathrm{p}$ dimensions using spaces of smaller dimensions. 


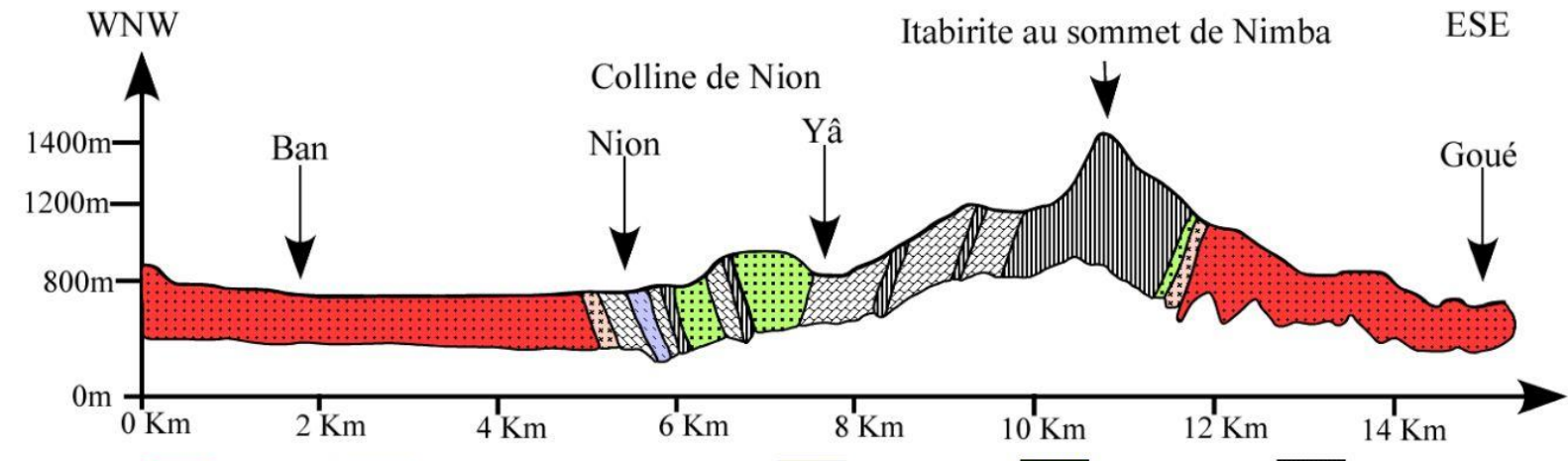

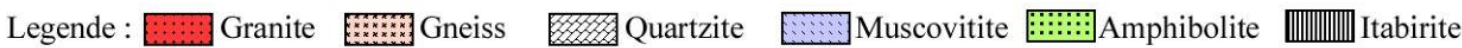

Figure 2: Cross section in the southern part of the Nimba region near the border between the Republic of Guinea and Liberia

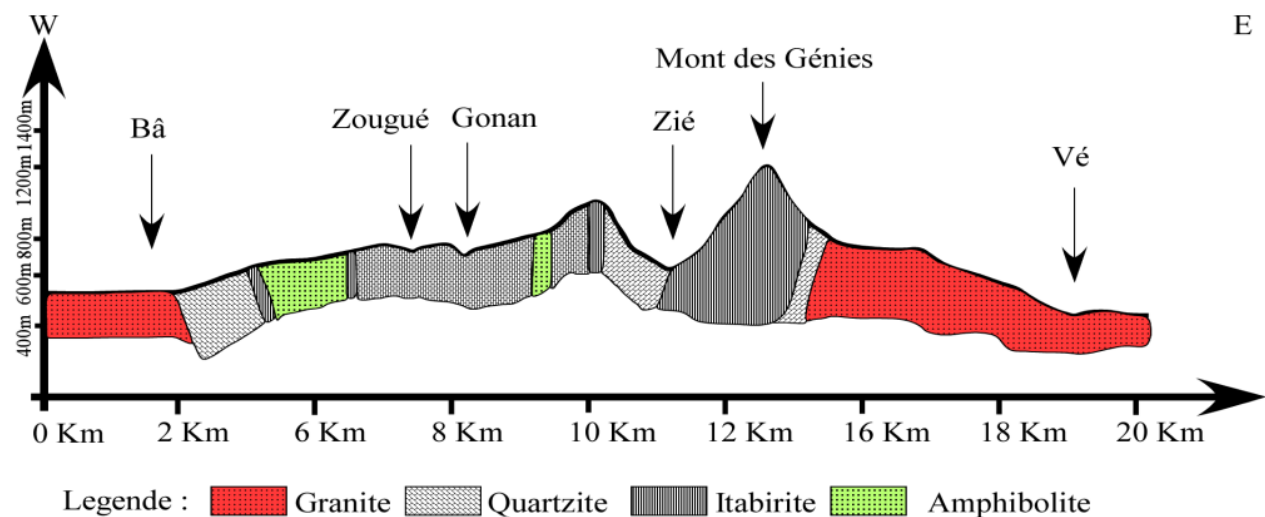

Figure 3: Cross section in the northern part of the Nimba region in the Republic of Guinea

\subsection{Petrographic Results}

Table 1: Mineralogical composition of the petrographic facies of Nion Hill (Nion District) and Mont des Génies (Gbié District)

\begin{tabular}{|c|c|c|c|c|c|c|c|}
\hline Minéraux & Gneiss & Gneiss à biotite & Granite & Quartzite & Muscovitite & Amphibolite & Itabirite \\
\hline Quartz & +++ & +++ & +++ & +++ & ++ & ++ & ++ \\
\hline Muscovite & + & + & ++ & +++ & +++ & + & + \\
\hline Biotite & ++ & +++ & ++ & & & + & \\
\hline $\begin{array}{l}\text { Feldspath } \\
\text { Potassique }\end{array}$ & +++ & +++ & +++ & +++ & + & ++ & ++ \\
\hline Plagioclase & +++ & +++ & +++ & + & & + & ++ \\
\hline Epidote & - & - & - & - & & ++ & \\
\hline Grunérite & - & - & - & - & & +++ & \\
\hline Microcline & ++ & ++ & ++ & - & & & \\
\hline Magnétite & - & - & - & + & & ++ & +++ \\
\hline Hématite & - & - & - & + & & & ++ \\
\hline Séricite & + & + & + & & & & \\
\hline Chlorite & & & + & & & & \\
\hline Oxyde & - & - & - & + & & & + \\
\hline Zircon & + & + & + & + & & & \\
\hline Apatite & + & + & + & & & & \\
\hline Opaques minerals & & & + & + & ++ & & \\
\hline
\end{tabular}


Macroscopic observation of rocks in the Nimba region

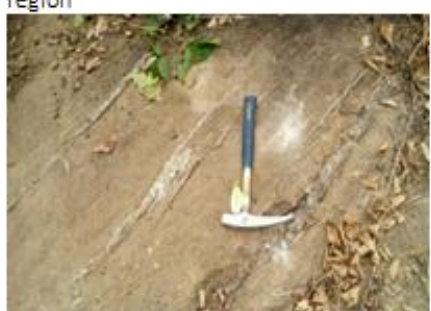

Photo 2a: Gneiss with quartz veins in the district of Nion ( $7^{\circ} 36^{\prime} 5.6916^{\prime \prime} \mathrm{N}-8^{\circ} 28^{\prime} 52.2480^{\prime \prime} \mathrm{W}$ ).

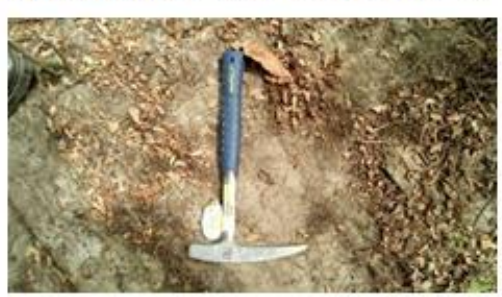

Photo 2d: Biotite gneiss in the Gbie district in the north of the Nimba range $\left(7^{\circ} 39^{\prime} 5.2174^{\prime \prime} \mathrm{N}\right.$ $8^{\circ} 19^{\prime} 58.3406^{\prime \prime} \mathrm{W}$

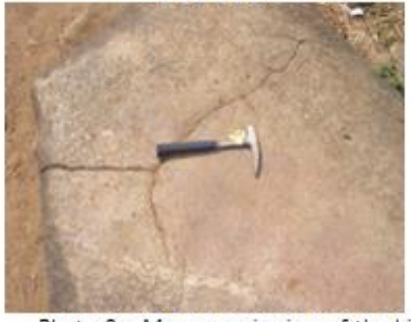

Photo $2 \mathrm{~g}$ : Macroscopic view of the biotite granite dome in the Gbié area $\left(7^{\circ} 40^{\prime} 18.1128\right.$ $\left.\mathrm{N}-8^{\circ} 18^{\prime} 57.3838^{\prime \prime} \mathrm{W}\right)$.

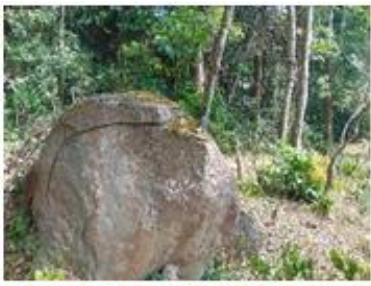

Photo 2j: Granite ball in the Nimba reserve $\left(7^{\circ}\right.$ $39^{\prime} 50.0329^{\prime \prime} \mathrm{N}-8^{\circ} 19^{\prime} 22.9908^{\prime \prime} \mathrm{W}$ )

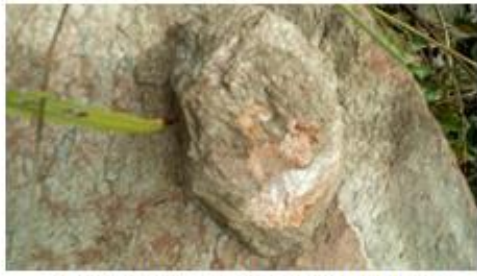

Photo $2 \mathrm{~m}$ : Muscovite at Nion Hill $\left(7^{\circ}\right.$ $\left.36^{\prime} 14.2163^{\prime \prime} \mathrm{N}-8^{\circ} 36^{\prime} 44.0830^{\prime \prime} \mathrm{W}\right)$

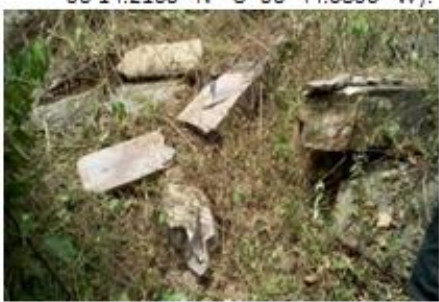

Photo 2p: Quartzite blocks (District of Nion) ( $7^{\circ}$ $36^{\prime} 14,544^{\prime \prime} \mathrm{N}-8^{\circ} 28^{\prime} 43,550^{\prime \prime} \mathrm{W}$

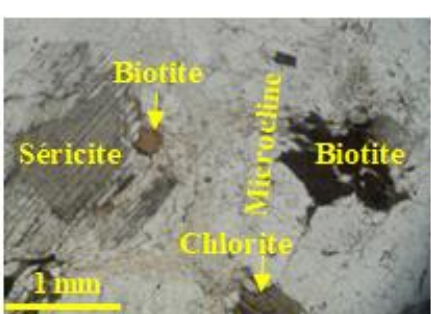

Photo 2b: Biotite inclusion in sericite, PNAL.

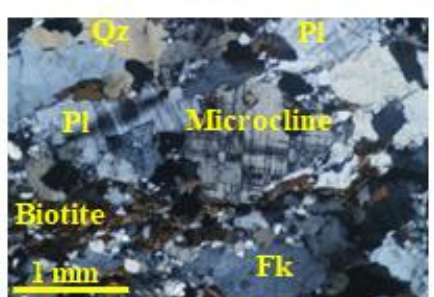

Photo 2e: Microline phenocrystal in contact with biotite, plagioclase (PI quartz $(\mathrm{Q} z$ ) and alkaline feldspar ( $\mathrm{Fk}$ ). $\mathrm{PAL}$.

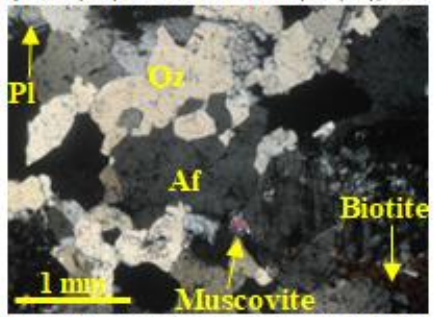

Photo $2 \mathrm{~h}$ : Inclusion of muscovite in alkali feldspar (Af) in PAL.

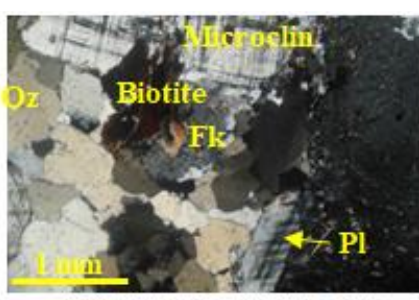

Photo $2 \mathrm{k}$ : Biotite surrounded by microcline, quartz $(Q z)$ and potash feldspar (Fk). Plagioclase (PI) in PAL.

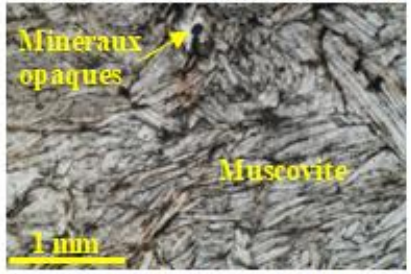

Photo $2 n$ : Inclusion of opaque minerals in muscovite, PNAL.

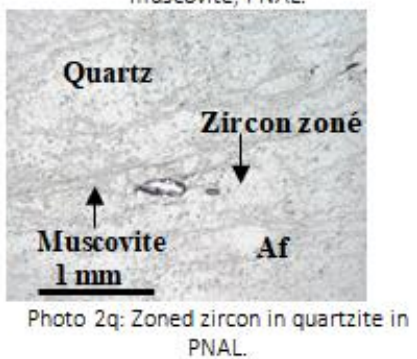

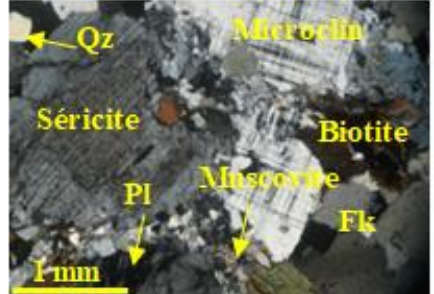

Photo $2 \mathrm{c}$ : Microcline and sericite phenocrysts in quartz $(\mathrm{Q} z)$, plagioclase $(\mathrm{PI})$, alkali feldspar $[\mathrm{Fk}\}$

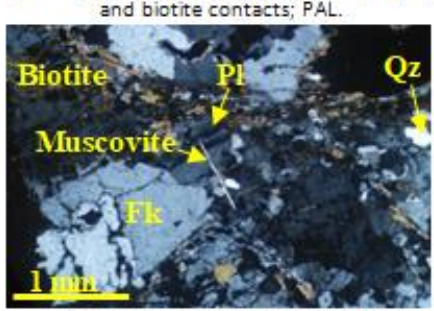

Photo 2f: Inclusion of muscovite in plagioclase (PI) and alkali feldspar ( $\mathrm{Fk}$ );

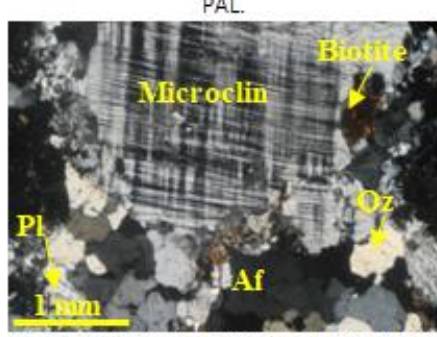

Photo 2i: Microcline surrounded by quart microcrystals $\left(\mathrm{O}_{2}\right)$, biotite, and alkali feldspar (Af) in PAL

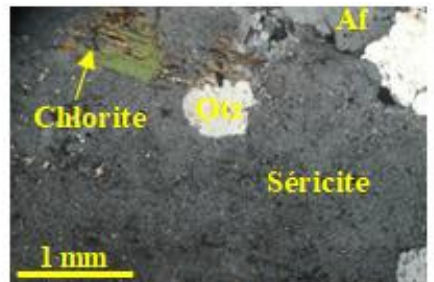

Photo 21: Inclusion of quartz ( $\mathrm{Qz}$ ) and chloritization of biotite in PAL sericite.

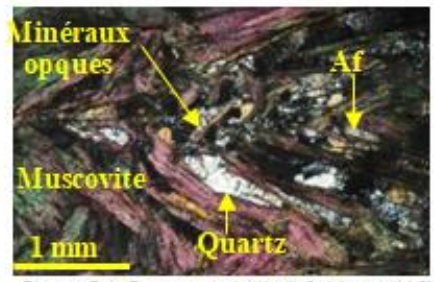

Photo 20: Quartz and alkali feldspar (Af) inclusions in muscovite, PAL.

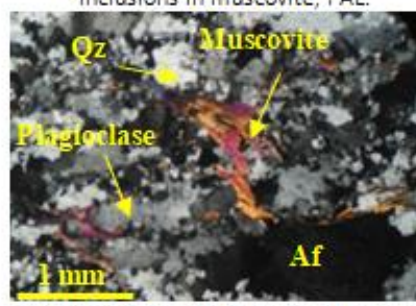

Photo $2 \mathrm{r}$ : "S" slit of muscovite, quartz $(\mathrm{Qz}$ ) and alkaline feldspar (Af) in " $\mathrm{C}$ ", hence "C $S^{\prime \prime}$, PAL. 


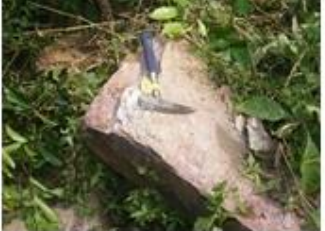

Photo 2s: Quartzite bench (District of Nion at 7 $36^{\prime} 137987^{\prime \prime} \mathrm{N}-8^{\circ} 28^{\prime} 44.2344^{\prime \prime}$ W).

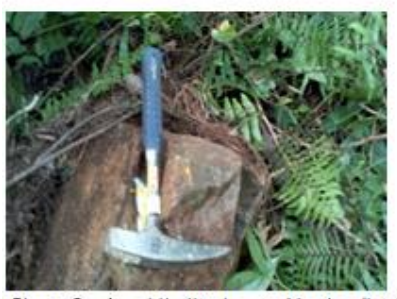

Photo 2v: Amphibolite located in the district of Nion $\left(7^{\circ} 37^{\prime} 28.4427^{\prime \prime} \mathrm{N}-8^{\circ} 26^{\prime} 6.34826^{\prime \prime} \mathrm{W}\right)$.

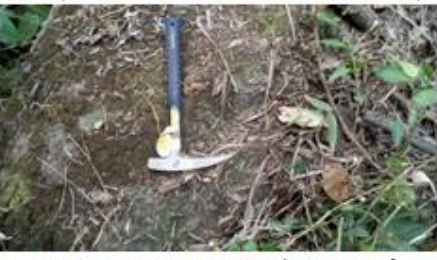

Photo $2 \%$ : Amphibolite in Gbié district $\left(7^{\circ} 40\right.$ 13.5072 " $\mathrm{N}-8^{\circ} 20^{\prime} 32.6385^{\prime \prime} \mathrm{W}$ ).

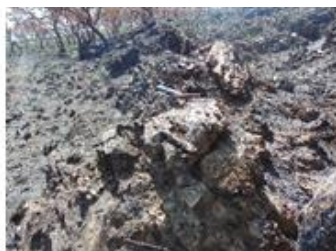

Photo 2ab: Alignment of itabirite benches with almost vertical dip (Nion District, $7^{\circ} 36^{\prime} 17.0828^{\prime \prime}$ $\mathrm{N}-8^{\circ} 25^{\prime} 50.5897^{\prime \prime}$ W)

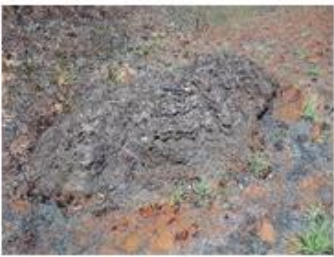

Photo 2ae: Itabirite micro-folds at the top of Mont des Génies (Gbié district, $7^{\circ} 39^{\prime} 44.7588^{\prime \prime}$ $\left.\mathrm{N}-8^{\circ} 21^{\prime} 25.5816^{\prime \prime} \mathrm{W}\right)$.

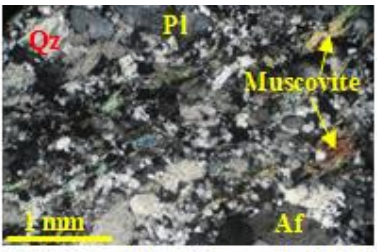

Photo 2t: Inclusion of muscovite in alka feldspar (Af), plagioclase and quartz,

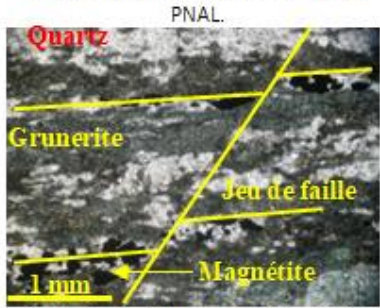

Photo 2w: fault in amphibolite, PNAL.

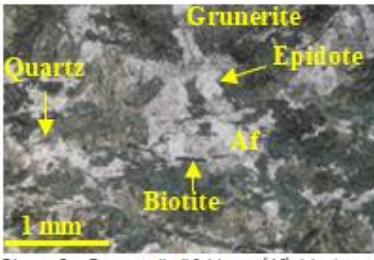

Photo 2r: Quartzalkalifeldspar (Af), biotite and epidote inclusions in grunerite, PNAL.

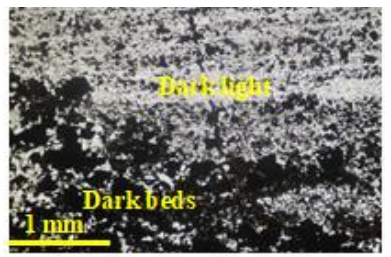

Photo 2ac: Alternating dark beds

(magnetite, sometimes hematite, pyrite) and light beds of quartz $(Q z)$, alkali

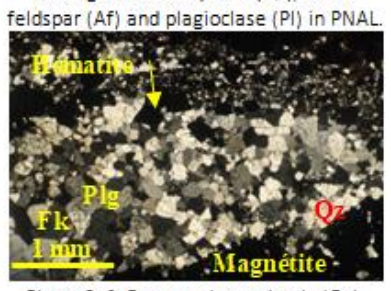

Photo 2af: Quartz microscristals (Qz), alkali feldspar (Af) and plagioclase ( $\mathrm{Plg}$ )

forming a white area surrounded by

magnetite and sometimes hematite and pyrite, PNAL.

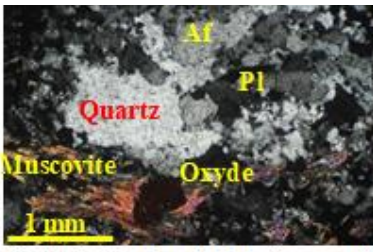

Photo 2u: Inclusion of oxide in muscovite in contact with alkali feldspar (Af), quart

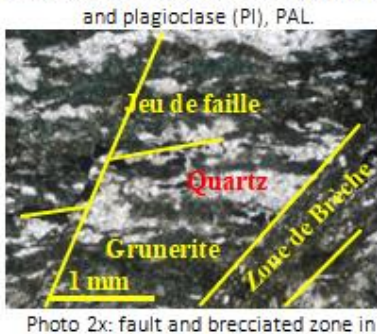

Photo 2x: fault and brecciated zone in amphibolite, PNAL.

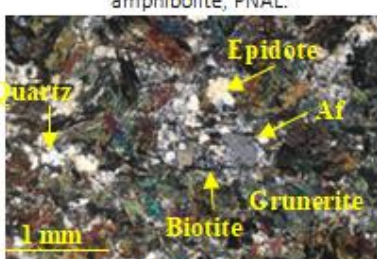

Photo 2a: Quartz, alkalifeldspar (Af), biotite and epidote inclusions in grunerite, PAL.

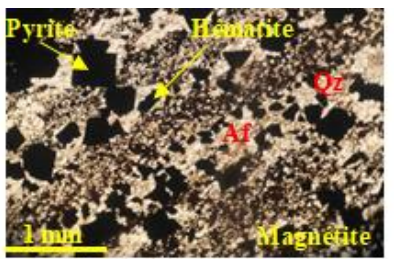

Photo 2ad: Alternating dark beds

(magnetite, sometimes hematite, pyrite) and light beds of quartz (Qz), alkali

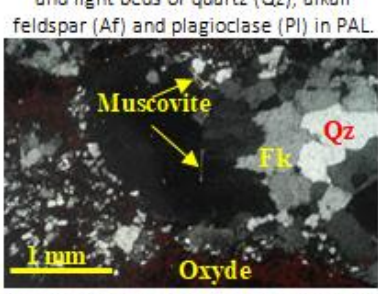

Photo 2 ag. Inclusion of muscovite in alkati laver at the contact with oxides in PAL.

Photo 2: Macroscopic and microscopic photographs of rocks in the Nimba region

\section{3-Results of the mineralogical study}

\subsubsection{The RAMAN}

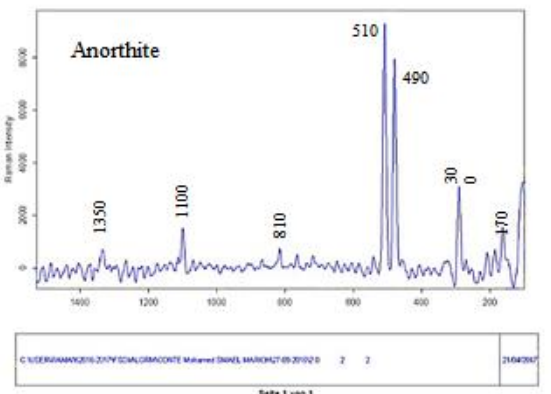

Figure 4a: Spectroscopy of gneisses [12]

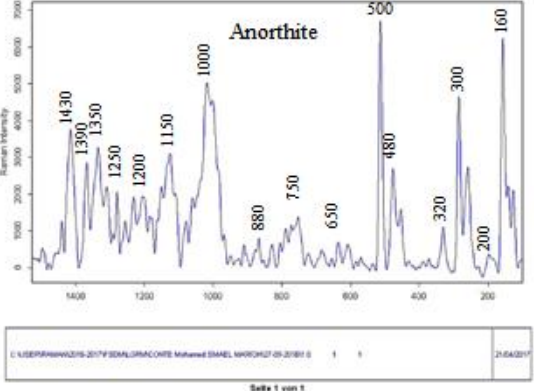

Figure 4b: Spectroscopy of granites [13]

261 | Int. J. of Multidisciplinary and Current research, Vol.6 (March/April 2018) 


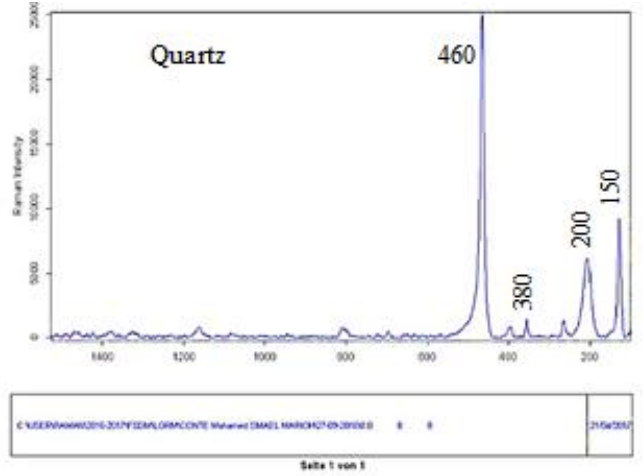

Figure 4c: Spectroscopy of quartzitic rocks [14]

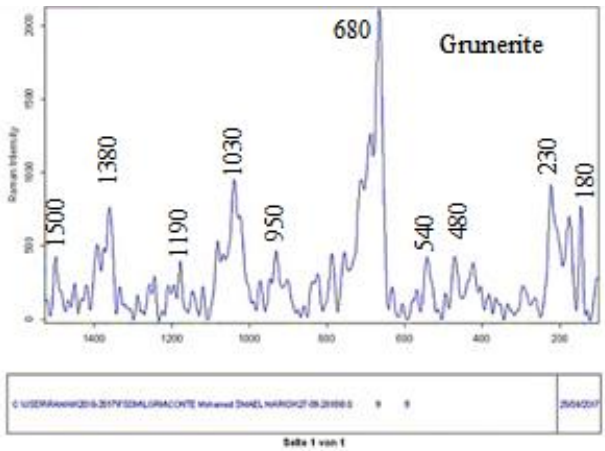

Figure 4e: spectroscopy of amphibolitic rocks [16]

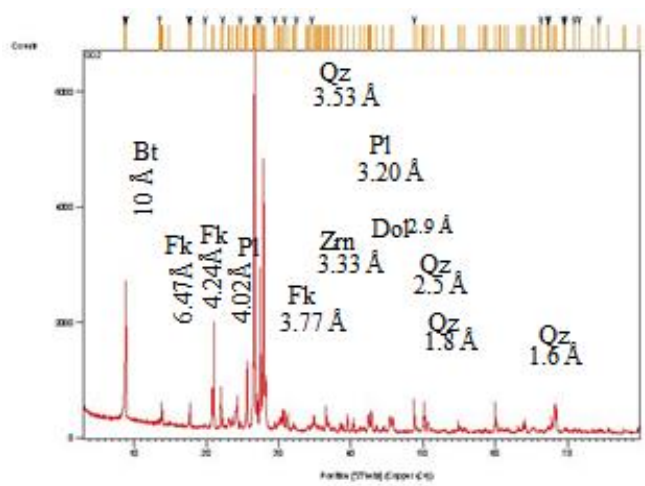

Figure 5a : Diffractogram of Gneiss: Biotite = 'Bt', Plagioclase $=$ 'PI', alkali Feldspar $=$ 'Af', Quartz = 'Qz', Zircon = 'Zrn', Anorthite = 'An', Dolomite = 'Dol'.

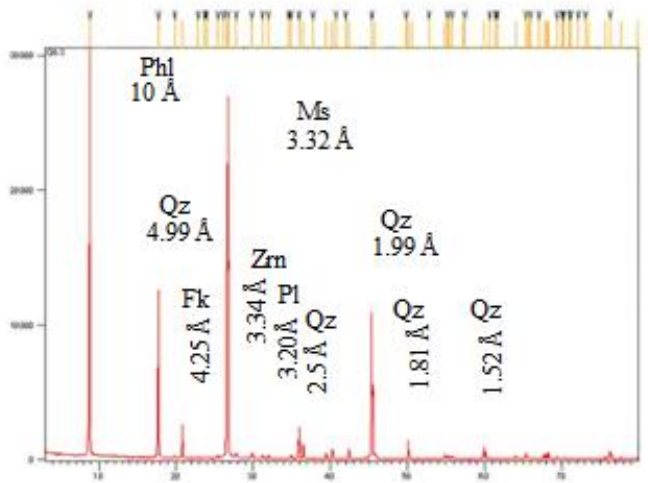

Figure 5c: Quartzite diffractogram: Phlogopite = 'Phl', Quartz = 'Qz', Potassium feldspar = 'Fk', Zircon = 'Zrn', Muscovite $=$ 'Ms', Plagioclase $={ }^{\prime} \mathrm{Pl}$ ' (Oligoclase).

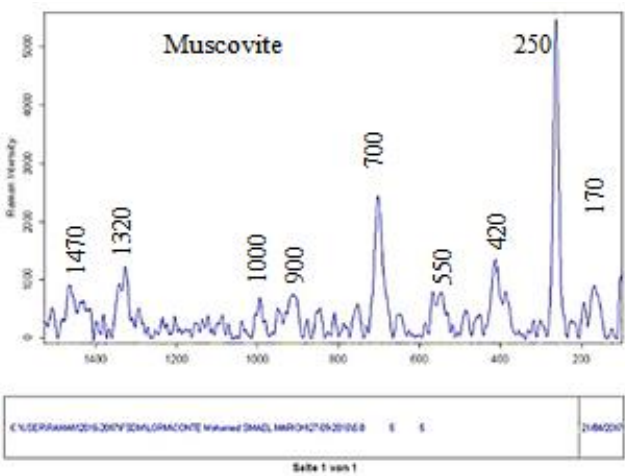

Figure 4d: Spectroscopy of muscovititic rocks [15]

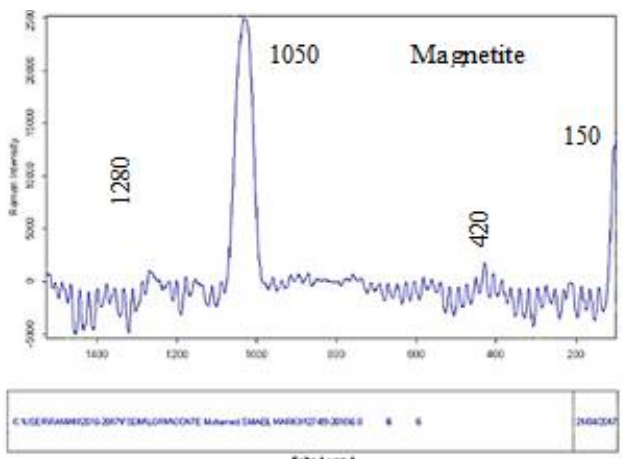

Figure 4f: spectroscopy of itabiritic rocks [17]

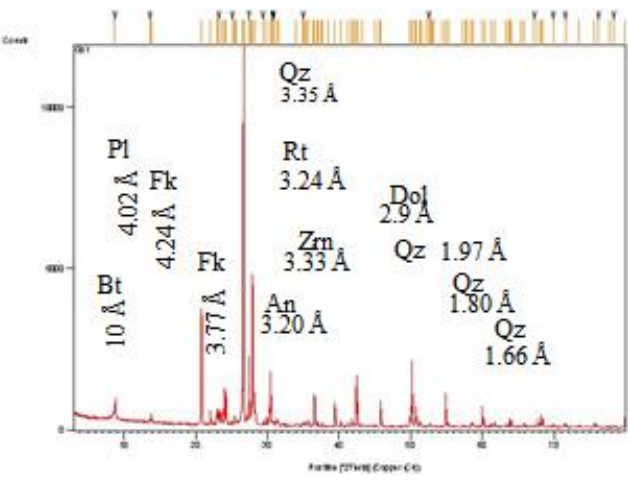

Figure 5b: Diffractogram of the granite:

Biotite $=$ 'Bt', Plagioclase $=$ 'Pl', alkali Feldspar = 'Af', Quartz = 'Qz', Zircon = 'Zrn', Rutile 'Rt', Anorthite $=$ 'An', Dolomite $=$ 'Dol'

cons

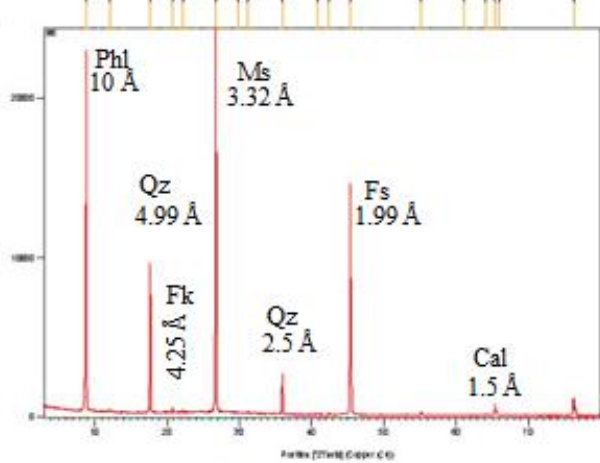

Figure 5d : Diffractogram of muscovitite: Phlogopite = 'Phl', Quartz = 'Qz', Potassium feldspar = 'Fk', Muscovite $=$ 'Ms', Fersilicite = 'Fs', Calcite $=$ 'Cal' 


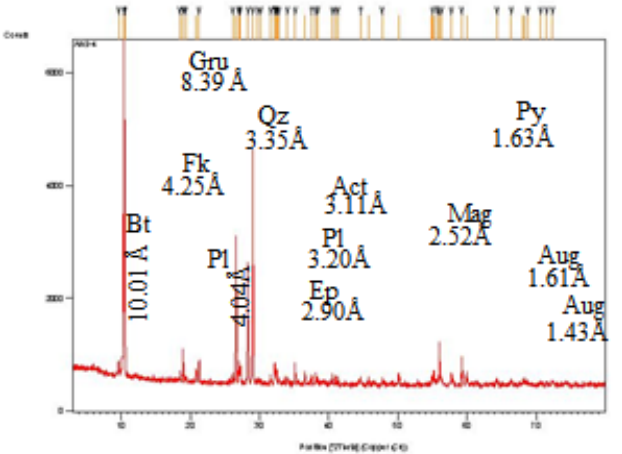

Figure 5e : Diffractogram of amphibolite: Biotite $=$ 'Bt', Grunerite = 'Gru', Potassium Feldspar = 'Fk', Plagioclase = 'PI', Quartz = 'Qz', Actinolite = 'Act', Plagioclase $=$ 'Pl' (Betowint), Epidote = 'Ep', Magnetite $=$ 'Mag', Pyrite $=$ 'Py', Augite = 'Aug'.

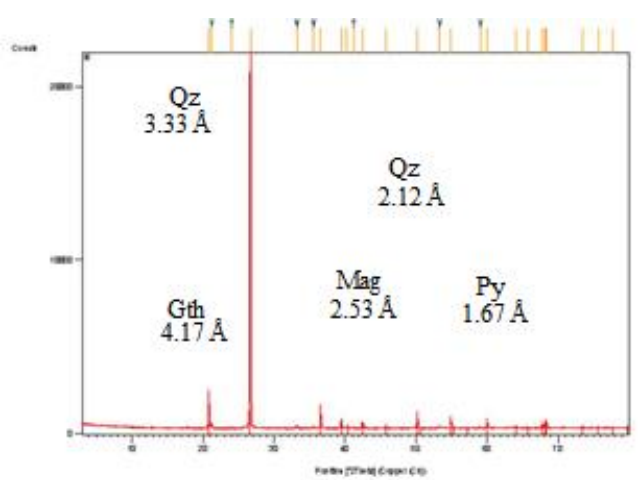

Figure $\mathbf{5 f}$ : Diffractogram of itabirite: Goethite = 'Gth', Quartz = 'Qz', Magnetite = 'Mag', Pyrite = 'Py'.

Figure 5: Diffractograms of rocks of the Nimba region [18]

Table 2: Mineralogy of different rocks in the Nimba region

\begin{tabular}{|c|c|}
\hline Samples & Minerals \\
\hline Gneiss & Biotite, Plagioclase (Anorthite), Potassium Feldspar, Quartz, Zircon, Dolomite. \\
\hline Granites & Biotite, Plagioclase (Anorthite), Potassium Feldspar, Quartz, Zircon, Rutile, Dolomite. \\
\hline Quartzites & Phlogopite, Quartz, Potassium Feldspar, Zircon, Muscovite, Plagioclase (Oligoclase). \\
\hline Muscovitites & Phlogopite, Quartz, Potassium Feldspar, Muscovite, Fersilicite. Calcite. \\
\hline Amphibolites & $\begin{array}{c}\text { Biotite, Grunerite, Potassium Feldspar, Plagioclase (Bytownite), Quartz, Actinolite, Epidote, } \\
\text { Magnetite, Pyrite, Augite. }\end{array}$ \\
\hline Itabirites & Goethite, Quartz, Magnetite, Pyrite. \\
\hline
\end{tabular}

\subsection{Geochemical results}

Table 3: Chemical analyzes of amphibolitic rocks in the Nimba region

\begin{tabular}{|c|c|c|c|c|c|c|c|c|c|}
\hline Echan & Amp01 & Amp02 & Amp03 & Amp04 & Amp05 & Amp06 & Amp07 & Amp08 & Amp09 \\
\hline $\mathrm{SiO} 2(\%)$ & 49,31 & 48.49 & 47.78 & 46.7 & 47.46 & 49.68 & 49.15 & 48.35 & 49.63 \\
\hline $\mathrm{TiO} 2$ & 1.05 & 1.08 & 0.57 & 1.21 & 1.82 & 1.29 & 0.94 & 0.89 & 0.81 \\
\hline $\mathrm{Al} 2 \mathrm{O} 3$ & 14.41 & 14.69 & 12.7 & 14.41 & 13.65 & 14.22 & 15.36 & 15.39 & 12.99 \\
\hline $\mathrm{Fe} 2 \mathrm{O} 3 \mathrm{t}$ & 14.45 & 13.69 & 11.54 & 13.35 & 17.17 & 15.49 & 12.51 & 12.65 & 10.54 \\
\hline $\mathrm{MnO}$ & 0.21 & 0.24 & 0.32 & 0.23 & 0.26 & 0.24 & 0.21 & 0.21 & 0.18 \\
\hline $\mathrm{MgO}$ & 6.88 & 6.05 & 13.14 & 9.01 & 5.25 & 6.53 & 8 & 7.9 & 9.85 \\
\hline $\mathrm{CaO}$ & 10.73 & 14.34 & 10.77 & 12.67 & 10.73 & 10.53 & 11.61 & 11.17 & 9.47 \\
\hline $\mathrm{Na} 2 \mathrm{O}$ & 2.32 & 1.11 & 1.48 & 1.42 & 2.07 & 0.67 & 1.89 & 2.25 & 2.46 \\
\hline $\mathrm{K} 2 \mathrm{O}$ & 0.24 & 0.27 & 0.54 & 0.14 & 0.56 & 0.61 & 0.13 & 0.28 & 1.63 \\
\hline P2O5 & 0.15 & 0.11 & 0.05 & 0.14 & 0.18 & 0.13 & 0.14 & 0.1 & 0.26 \\
\hline PF & 0.13 & 0.41 & 1.08 & 0.44 & 0.75 & 0.64 & 0.22 & 0.4 & 0.87 \\
\hline Tot & 99.88 & 100.44 & 99.97 & 99.72 & 99.91 & 100.03 & 99.72 & 99.59 & 98.69 \\
\hline $\mathrm{U}(\mathrm{ppm})$ & 0.22 & 0.18 & 0.12 & 0.07 & 0.49 & 0.1 & 0.1 & 0.1 & 0.3 \\
\hline Th & 0.81 & 0.93 & 0.22 & 0.22 & 0.52 & 0.46 & 0.51 & 0.46 & 1.01 \\
\hline $\mathrm{Ta}$ & 0.21 & 0.2 & 0.09 & 0.18 & 0.41 & 0.28 & 0.22 & 0.18 & 0.21 \\
\hline $\mathrm{Nb}$ & 2.86 & 2.74 & 1.11 & 2.61 & 5.13 & 3.65 & 2.79 & 2.3 & 2.094 \\
\hline $\mathrm{Hf}$ & 2.01 & 1.9 & 0.82 & 1.15 & 3.11 & 2.26 & 1.58 & 1.45 & 1.64 \\
\hline $\mathrm{Zr}$ & 70.68 & 72.95 & 30.42 & 32.79 & 115.4 & 83.05 & 59.5 & 52.3 & 62.11 \\
\hline $\mathrm{Y}$ & 23.78 & 24.03 & 12.66 & 18.63 & 39.22 & 26.96 & 20.86 & 18.67 & 16.67 \\
\hline $\mathrm{Ni}$ & 124.89 & 137.47 & 309.23 & 158.23 & 87.08 & 78.17 & 146.06 & 160 & 150 \\
\hline $\mathrm{Cr}$ & 206.74 & 255.27 & 822.95 & 32.44 & 148.29 & 217.56 & 316.01 & 316.87 & 660.36 \\
\hline Co & 54.44 & 53.57 & 63.21 & 53.04 & 54 & 50.79 & 51.76 & 54.6 & 43.1 \\
\hline $\mathrm{V}$ & 328.66 & 331.27 & 221.58 & 217.55 & 430.33 & 342.95 & 258.97 & 277.94 & 233.51 \\
\hline La & 4.67 & 4.51 & 1.64 & 2.18 & 6.4 & 5.11 & 3.87 & 3.17 & 8.86 \\
\hline $\mathrm{Ce}$ & 11.85 & 10.95 & 4.28 & 7.14 & 17.13 & 13.42 & 10.28 & 8.96 & 21.21 \\
\hline $\mathrm{Pr}$ & 1.66 & 1.62 & 0.67 & 1.23 & 2.66 & 1.97 & 1.59 & 1.31 & 2.96 \\
\hline $\mathrm{Nd}$ & 8.14 & 7.99 & 3.58 & 6.81 & 13.34 & 9.99 & 6.92 & 6.54 & 13.46 \\
\hline $\mathrm{Sm}$ & 2.65 & 2.63 & 1.28 & 2.5 & 4.43 & 3.13 & 2.23 & 2.07 & 3.26 \\
\hline $\mathrm{Eu}$ & 0.87 & 0.96 & 0.54 & 0.9 & 1.32 & 1.11 & 0.77 & 0.75 & 1 \\
\hline
\end{tabular}




\begin{tabular}{|c|c|c|c|c|c|c|c|c|c|}
\hline $\mathrm{Gd}$ & 2.94 & 3.41 & 1.6 & 3.18 & 4.84 & 4.01 & 2.54 & 2.73 & 3.05 \\
\hline $\mathrm{Tb}$ & 0.53 & 0.59 & 0.28 & 0.51 & 0.88 & 0.64 & 0.46 & 0.44 & 0.42 \\
\hline Dy & 3.83 & 3.84 & 1.81 & 3.23 & 5.92 & 4.28 & 3.02 & 2.99 & 2.85 \\
\hline $\mathrm{Ho}$ & 0.83 & 0.88 & 0.43 & 0.7 & 1.37 & 0.95 & 0.7 & 0.66 & 0.6 \\
\hline $\mathrm{Er}$ & 2.09 & 2.27 & 1.17 & 1.74 & 3.62 & 2.61 & 1.93 & 1.97 & 1.63 \\
\hline $\mathrm{Tm}$ & 0.32 & 0.36 & 0.2 & 0.24 & 0.63 & 0.46 & 0.31 & 0.28 & 0.27 \\
\hline $\mathrm{Yb}$ & 2.3 & 2.38 & 1.32 & 1.71 & 4.13 & 2.44 & 1.93 & 2.11 & 1.15 \\
\hline $\mathrm{Lu}$ & 0.36 & 0.38 & 0.22 & 0.24 & 0.63 & 0.39 & 0.31 & 0.323 & 0.26 \\
\hline $\mathrm{Li}$ & 9 & 5 & 13 & 8 & 12 & 12 & 7 & 17 & 21 \\
\hline Cs & 0.11 & 0.71 & 0.4 & 0.41 & 0.25 & 0.14 & 0.1 & 0.1 & 1.4 \\
\hline $\mathrm{Rb}$ & 6.5 & 8.84 & 24.91 & 5.61 & 13.94 & 16.7 & 3.84 & 5.86 & 75.5 \\
\hline $\mathrm{Ba}$ & 58.11 & 30.14 & 384.73 & 60.6 & 151.25 & 54.77 & 36.14 & 53.26 & 366.99 \\
\hline $\mathrm{Sr}$ & 93.34 & 116.98 & 64.61 & 161.02 & 105.9 & 90.96 & 103.29 & 108.62 & 373.47 \\
\hline As & 0.51 & 0.18 & 0.52 & 0.3 & 0.42 & 1.47 & 0.31 & 0.51 & 0.89 \\
\hline $\mathrm{Be}$ & 0.32 & 0.44 & 0.13 & 0 & 1.16 & 0 & 0.61 & 0.41 & 0.47 \\
\hline $\mathrm{Bi}$ & 0.03 & 0.07 & 0.15 & 0.02 & 0.17 & 0.08 & 0.02 & 0.22 & 0.11 \\
\hline $\mathrm{Cd}$ & 0.26 & 0.08 & 0.05 & 0.09 & 0.22 & 0.18 & 0.16 & 0 & 0.02 \\
\hline $\mathrm{Cu}$ & 121.64 & 100.29 & 60.16 & 124.13 & 63.74 & 67.18 & 94.56 & 80.01 & 10.8 \\
\hline $\mathrm{Ga}$ & 19.68 & 19.26 & 12.34 & 12.34 & 21.14 & 19.29 & 17.55 & 17.89 & 15.83 \\
\hline $\mathrm{Ge}$ & 1.28 & 1.58 & 1.37 & 1.31 & 1.18 & 147 & 1.4 & 1.51 & 1.26 \\
\hline In & 0.07 & 0.07 & 0.06 & 0.07 & 0.1 & 0.07 & 0.05 & 0.07 & 0.04 \\
\hline Mo & 0.76 & 1.22 & 0.36 & 0.45 & 0.6 & 0.86 & 0.92 & 1.19 & 0.69 \\
\hline $\mathrm{Pb}$ & 2.99 & 2.27 & 2.26 & 1.11 & 3.01 & 1.67 & 0.79 & 4.45 & 4.96 \\
\hline $\mathrm{Sb}$ & 0.11 & 0.19 & 0.09 & 0.12 & 0.07 & 0.4 & 0.04 & 0.19 & 0.1 \\
\hline Sn & 1.19 & 0.81 & 0.55 & 0.22 & 1.63 & 0.84 & 0.65 & 0.74 & 0.77 \\
\hline$W$ & 0.21 & 0.22 & 0.36 & 0.14 & 0.67 & 0.2 & 0.13 & 0.47 & 0.43 \\
\hline $\mathrm{Zn}$ & 112.88 & 102.37 & 71.26 & 88.94 & 154.48 & 115.22 & 90.25 & 102.23 & 86.94 \\
\hline
\end{tabular}

Table 4: Chemical analyzes of granitic rocks in the Nimba region

\begin{tabular}{|c|c|c|c|c|c|c|c|c|c|}
\hline Echan & Gr01 & Gr02 & Gr03 & Gr04 & Gr05 & Gr06 & Gr07 & Gr08 & Gr09 \\
\hline $\mathrm{SiO} 2$ & 73.22 & 73.63 & 74.1 & 70.79 & 66.64 & 67.9 & 73.17 & 73.52 & 74.89 \\
\hline TiO2 & 0.02 & 0.22 & 0.11 & 0.27 & 0.71 & 0.35 & 0.19 & 0.14 & 0.08 \\
\hline $\mathrm{Al} 203$ & 14.73 & 13.75 & 13.87 & 15.19 & 15.52 & 14.7 & 14.06 & 14.51 & 13.77 \\
\hline $\mathrm{Fe} 2 \mathrm{O} 3 \mathrm{t}$ & 0.88 & 2.18 & 1.14 & 1.7 & 4.94 & 4.24 & 1.17 & 1.4 & 0.95 \\
\hline $\mathrm{MnO}$ & 0.03 & 0.02 & 0.04 & 0.03 & 0.07 & 0.06 & 0.04 & 0.02 & 0.04 \\
\hline $\mathrm{MgO}$ & 0.21 & 0.31 & 0.17 & 0.45 & 1.59 & 0.9 & 0.26 & 0.25 & 0.16 \\
\hline $\mathrm{CaO}$ & 1.45 & 1.24 & 1.26 & 1.74 & 2.53 & 2.3 & 1.38 & 1.59 & 0.83 \\
\hline $\mathrm{Na} 2 \mathrm{O}$ & 3.03 & 2.4 & 3.01 & 4.21 & 4.63 & 4 & 3.67 & 4.06 & 3.53 \\
\hline $\mathrm{K} 2 \mathrm{O}$ & 5.97 & 5.9 & 5.37 & 3.76 & 1.79 & 3.27 & 4.42 & 3.75 & 4.98 \\
\hline $\mathrm{P} 2 \mathrm{O} 5$ & 0.05 & 0.05 & 0.04 & 0.13 & 0.23 & 0.19 & 0.09 & 0.11 & 0.1 \\
\hline $\mathrm{PF}$ & 0.3 & 0.47 & 0.19 & 0.75 & 1.02 & 1 & 0.55 & 0.44 & 0.3 \\
\hline Tot & 99.89 & 99.9 & 99.57 & 99.02 & 99.67 & 98.91 & 99.54 & 99.79 & 99.63 \\
\hline $\mathrm{U}(\mathrm{ppm})$ & 22.15 & 5.05 & 12.16 & 2.43 & 1.8 & 1.9 & 9.76 & 0.41 & 8.63 \\
\hline Th & 18.37 & 94.8 & 63.29 & 20.78 & 18.25 & 34.7 & 32.22 & 13.35 & 30.48 \\
\hline $\mathrm{Ta}$ & 0.06 & 0.23 & 0.16 & 0.17 & 0.72 & 0.9 & 1.73 & 0.03 & 0.86 \\
\hline $\mathrm{Nb}$ & 0.97 & 10.6 & 3.33 & 4.26 & 10.77 & 13.3 & 14.45 & 1.21 & 7.17 \\
\hline $\mathrm{Hf}$ & 1.08 & 6.24 & 3.6 & 3.58 & 3.38 & 11.1 & 4.73 & 2.98 & 3.37 \\
\hline $\mathrm{Zr}$ & 33.56 & 223.5 & 122.75 & 155.11 & 142.34 & 377 & 177.27 & 125.46 & 96.8 \\
\hline$Y$ & 4.24 & 8.21 & 7.94 & 3.19 & 56.56 & 15.5 & 16.69 & 2.21 & 25.74 \\
\hline $\mathrm{Ni}$ & 4.6 & 4.06 & 3.63 & 2.25 & 17.51 & 10 & 3.7 & 3.05 & 2.57 \\
\hline $\mathrm{Cr}$ & 53.17 & 27.39 & 34.49 & 144 & 25.35 & 48 & 34.11 & 32.03 & 17.39 \\
\hline Co & 1.79 & 2.45 & 1.54 & 2.29 & 9.24 & 9 & 2.04 & 2.25 & 1.15 \\
\hline $\mathrm{V}$ & 2.24 & 2.24 & 11.03 & 3.47 & 9.42 & 46.36 & 47 & 7.24 & 1.02 \\
\hline La & 19.57 & 102.37 & 64.03 & 39.09 & 159.27 & 125 & 44.25 & 39.41 & 33.7 \\
\hline $\mathrm{Ce}$ & 37.62 & 178.46 & 90.88 & 64.39 & 190.12 & 219.3 & 76.92 & 61.61 & 61.17 \\
\hline $\mathrm{Pr}$ & 4.42 & 18.17 & 10.54 & 6.21 & 27.48 & 21.9 & 7.8 & 5.75 & 6.18 \\
\hline $\mathrm{Nd}$ & 15.37 & 59.42 & 33.57 & 20.35 & 92.33 & 73.1 & 25.55 & 17.63 & 21.18 \\
\hline $\mathrm{Sm}$ & 2.96 & 8.84 & 5.02 & 2.5 & 14.24 & 10.1 & 4.4 & 2.3 & 4.7 \\
\hline $\mathrm{Eu}$ & 0.99 & 0.9 & 0.9 & 0.92 & 2.19 & 1 & 0.93 & 0.99 & 0.07 \\
\hline $\mathrm{Gd}$ & 2.27 & 5.98 & 3.53 & 1.54 & 12.04 & 6.6 & 3.19 & 1.33 & 4.69 \\
\hline $\mathrm{Tb}$ & 0.27 & 0.68 & 0.37 & 0.19 & 1.72 & 0.9 & 0.46 & 0.16 & 0.76 \\
\hline
\end{tabular}




\begin{tabular}{|c|c|c|c|c|c|c|c|c|c|}
\hline $\mathrm{Dy}$ & 1.19 & 2.56 & 1.68 & 0.76 & 9.73 & 3.7 & 2.54 & 0.71 & 4.96 \\
\hline $\mathrm{Ho}$ & 0.16 & 0.23 & 0.24 & 0.11 & 1.82 & 0.7 & 0.51 & 0.09 & 1.14 \\
\hline $\mathrm{Er}$ & 0.29 & 0.88 & 0.64 & 0.3 & 4.82 & 1.9 & 1.47 & 0.21 & 2.8 \\
\hline $\mathrm{Tm}$ & 0.03 & 0.07 & 0.07 & 0.04 & 0.6 & 0.2 & 0.25 & 0.02 & 0.46 \\
\hline $\mathrm{Yb}$ & 0.125 & 0.44 & 0.44 & 0.23 & 3.27 & 1.5 & 1.46 & 0.15 & 2.76 \\
\hline $\mathrm{Lu}$ & 0.02 & 0.08 & 0.08 & 0.03 & 0.4 & 0.2 & 0.23 & 0.03 & 0.41 \\
\hline $\mathrm{Li}$ & 9 & 24 & 11 & 19 & 30 & 13 & 29 & 6 & 12 \\
\hline $\mathrm{Cs}$ & 0.74 & 0.65 & 0.75 & 0.56 & 0.8 & & 1.71 & 0.1 & 1.03 \\
\hline $\mathrm{Rb}$ & 128.89 & 239.93 & 206.23 & 117.86 & 94.85 & 86 & 207.72 & 57.23 & 158.02 \\
\hline $\mathrm{Ba}$ & 764.89 & 901.69 & 696.66 & 1300.55 & 317.6 & 202.4 & 1499.31 & 1852.58 & 760.14 \\
\hline $\mathrm{Sr}$ & 253.2 & 155.8 & 163.7 & 461.47 & 301.14 & 391 & 139.14 & 487.03 & 85.23 \\
\hline $\mathrm{As}$ & 0.25 & 0.09 & 0.24 & 0.25 & 0.1 & & 0.19 & 0.13 & 0.03 \\
\hline $\mathrm{Be}$ & 1.13 & 0 & 0.55 & 1.1 & 1.04 & & 2.16 & 0.36 & 1.25 \\
\hline $\mathrm{Bi}$ & 0.04 & 0.02 & 0.03 & 0.02 & 0 & & 0.02 & 0 & 0.01 \\
\hline $\mathrm{Cd}$ & 0.06 & 0.11 & 0.13 & 0.11 & 0.09 & & 00 & 0 & 0.01 \\
\hline $\mathrm{Cu}$ & 61.62 & 3.29 & 4.86 & 3.52 & 42.52 & & 2.69 & 4.5 & 2.87 \\
\hline $\mathrm{Ga}$ & 15.04 & 18.86 & 16.17 & 19.51 & 23.24 & & 20.73 & 17.4 & 18.42 \\
\hline $\mathrm{Ge}$ & 0.94 & 0.74 & 0.66 & 0.65 & 0.8 & & 0.85 & 0.53 & 0.86 \\
\hline $\mathrm{In}$ & 0.02 & 0.02 & 0.02 & 0.04 & 0.02 & & 0.05 & 0.02 & 0.01 \\
\hline $\mathrm{Mo}$ & 2.06 & 1.96 & 1.66 & 1.38 & 1.02 & & 2.23 & 1.23 & 1.3 \\
\hline $\mathrm{Pb}$ & 47.17 & 41.27 & 44.33 & 32.81 & 12.77 & & 33 & 16.12 & 44.04 \\
\hline $\mathrm{Sb}$ & 0.02 & 0.02 & 0.04 & 0.04 & 0.02 & & 0.05 & 0.04 & 0.03 \\
\hline $\mathrm{Sn}$ & 0.34 & 0.18 & 0.42 & 0.45 & 1.56 & & 1.91 & 0.29 & 0.75 \\
\hline $\mathrm{W}$ & 0.12 & 0.07 & 0.13 & 0.05 & 0.13 & & 0.26 & 0.13 & 0.18 \\
\hline $\mathrm{Zn}$ & 9.08 & 28.92 & 20.53 & 42.15 & 85.67 & & 35.3 & 24.86 & 24.02 \\
\hline
\end{tabular}

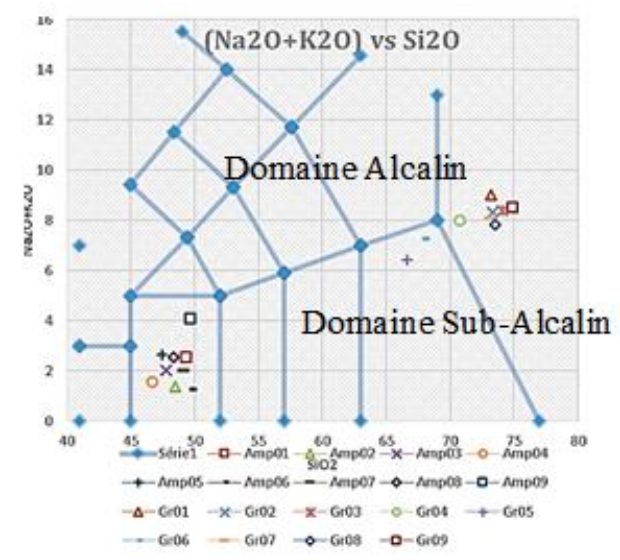

Figure 6: Projection of amphibolitic and granitic rocks from the Nimba range on the $\mathrm{Na} 2 \mathrm{O}+\mathrm{K} 2 \mathrm{O}$ vs. Si2O plot of TAS [22]

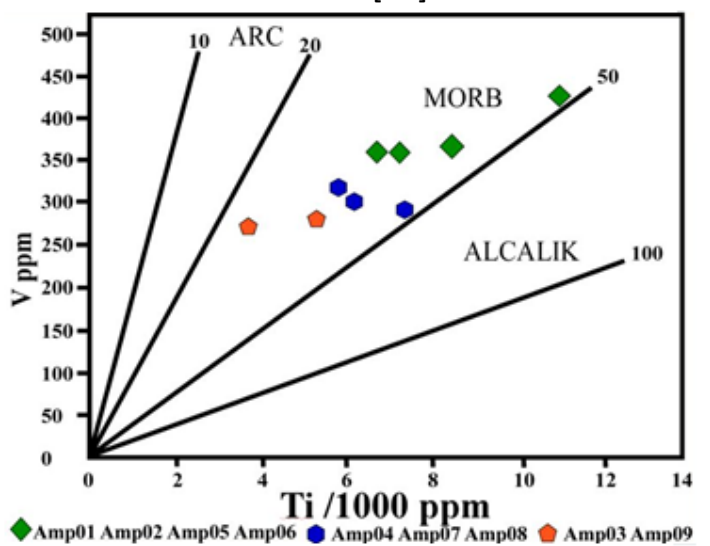

Figure 8: Distribution of amphibolitic rocks from the Nimba range in the $\mathrm{V}$ versus $\mathrm{Ti} / 1000$ diagram [24].

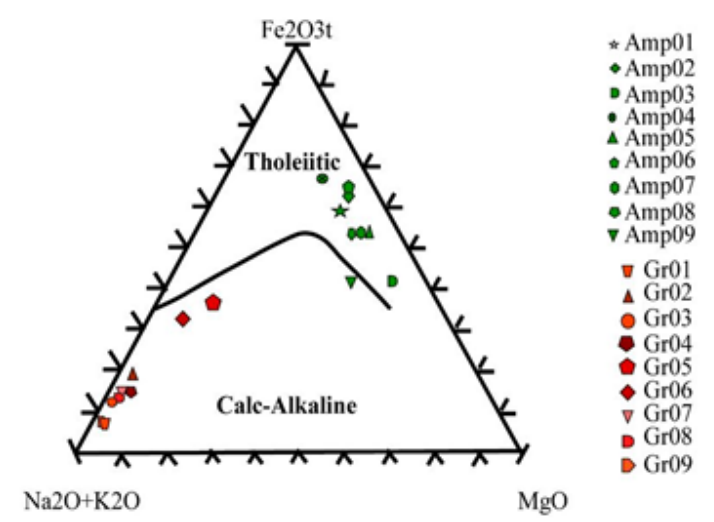

Figure 7: Placement of amphibolitic and granitic rocks of the Nimba range on the AFM diagram [23].

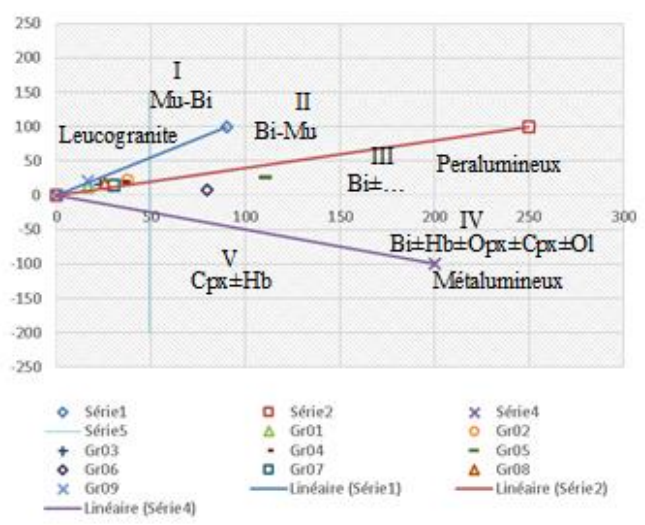

Figure 9: Projection of granitic rocks from the Nimba range on Diagram A versus B of Debon et Le Fort [25]. 


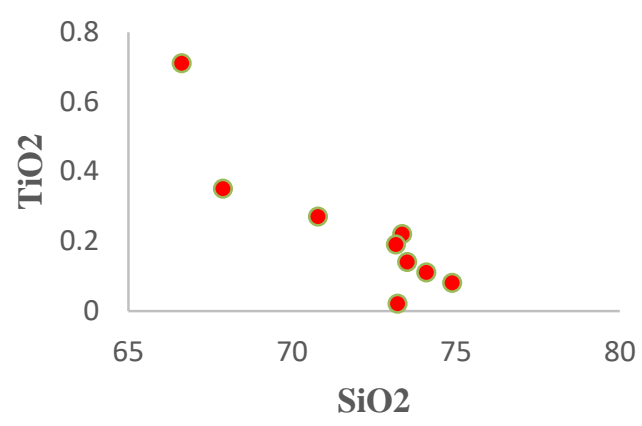

- Gr01 Gr02 Gr03 Gr04 Gr05 Gr06 Gr07 Gr08 Gr09

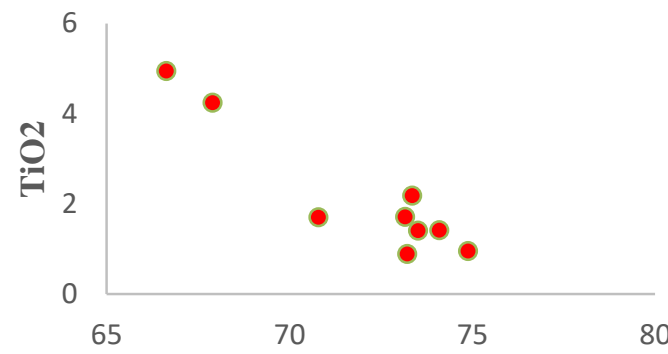

- Gr01 Gr02 Gr03 Gr04SiPß2 Gr06 Gr07 Gr08 Gr09
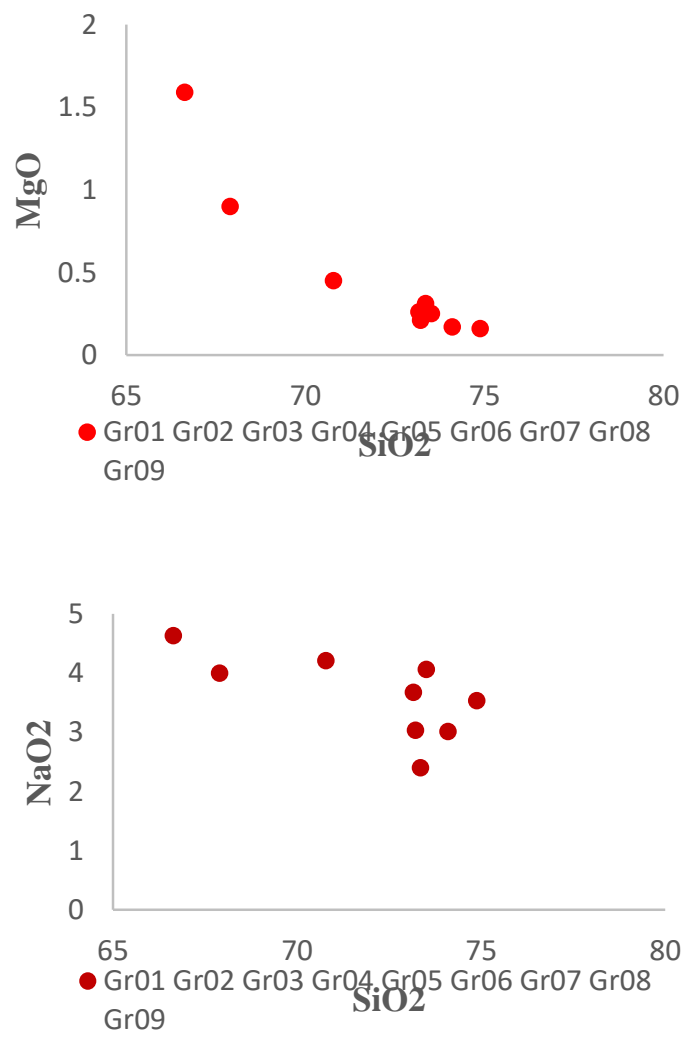

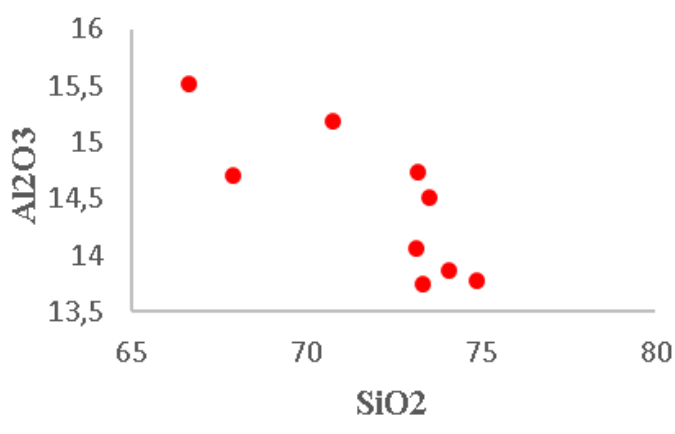

- Gr01 Gr02 Gr03 Gr04 Gr05 Gr06 Gr07 Gr08 Gr09
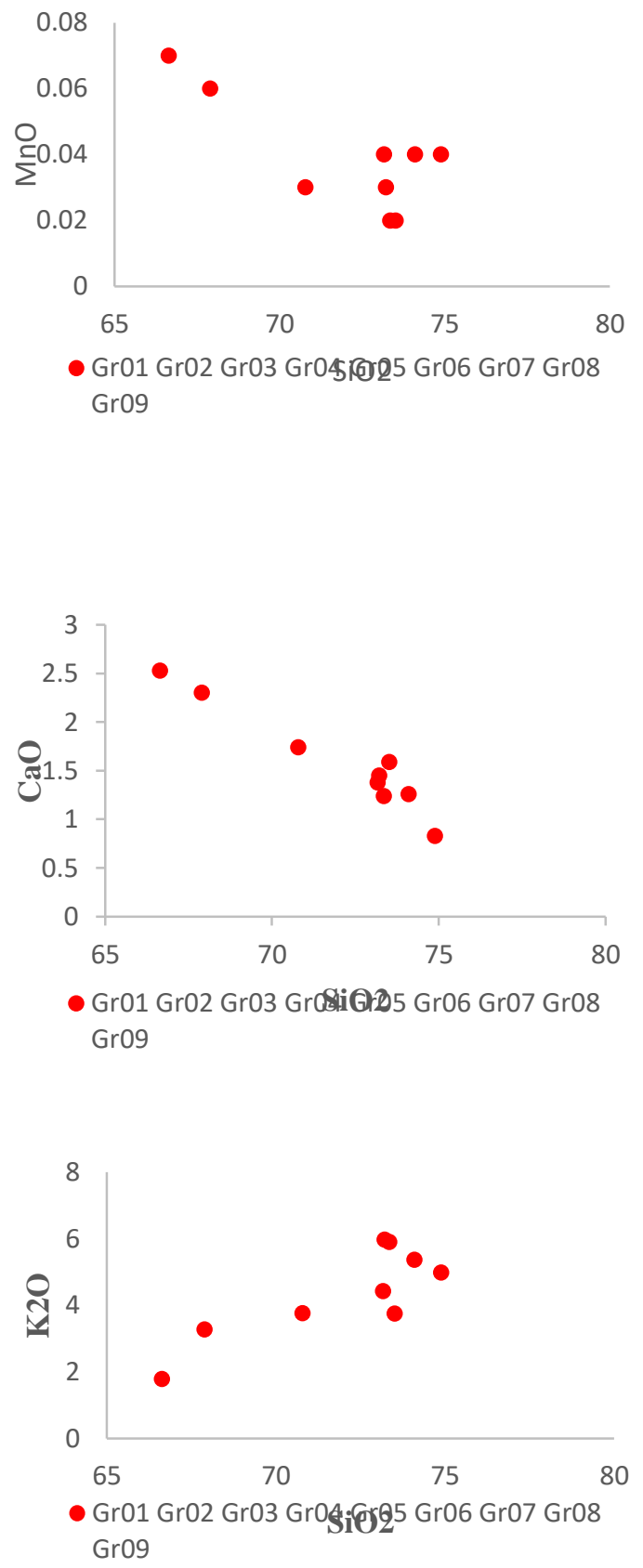


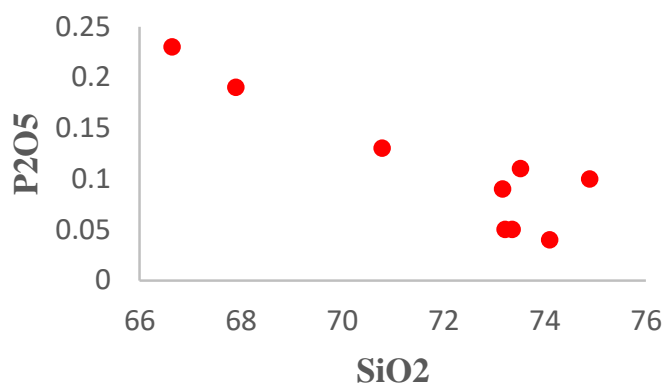

- Gr01 Gr02 Gr03 Gr04 Gr05 Gr06 Gr07 Gr08 Gr09

Figure 10: Harker diagrams of granitic rocks in the Nimba region.

10

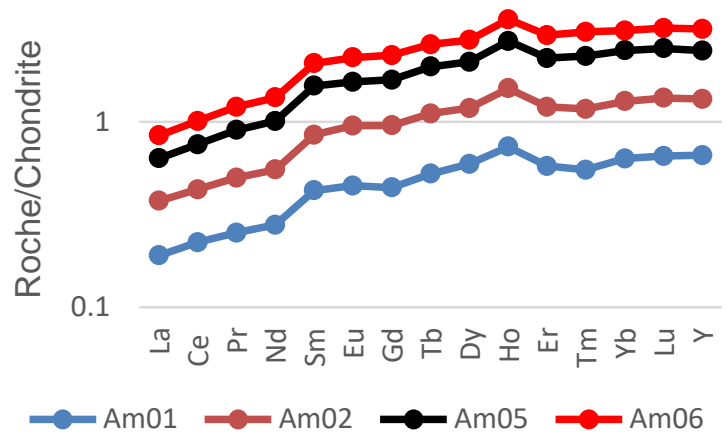

10

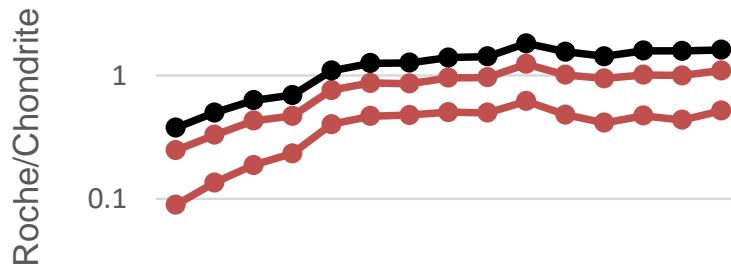

0.01

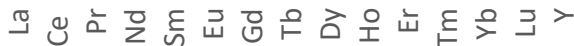

$\longrightarrow \mathrm{Am04} \longrightarrow \mathrm{Am07} \longrightarrow \mathrm{Am08}$

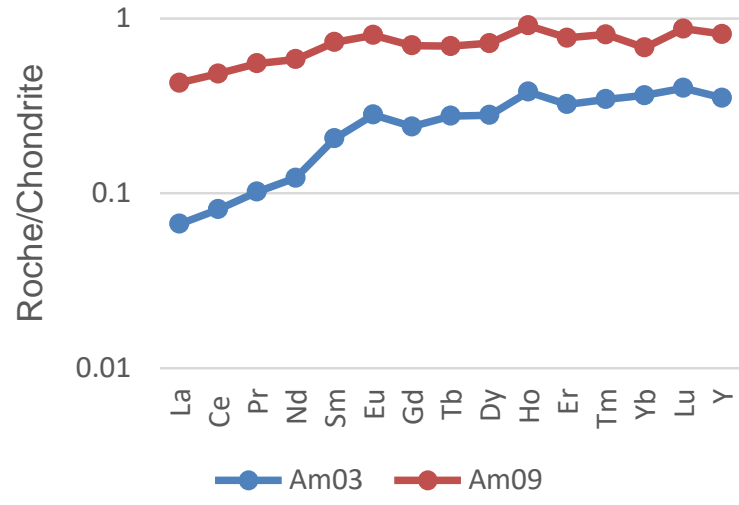

Figure 11: REE spectra normalized to chondrites of amphibolitic rocks of the Nimba range [26].

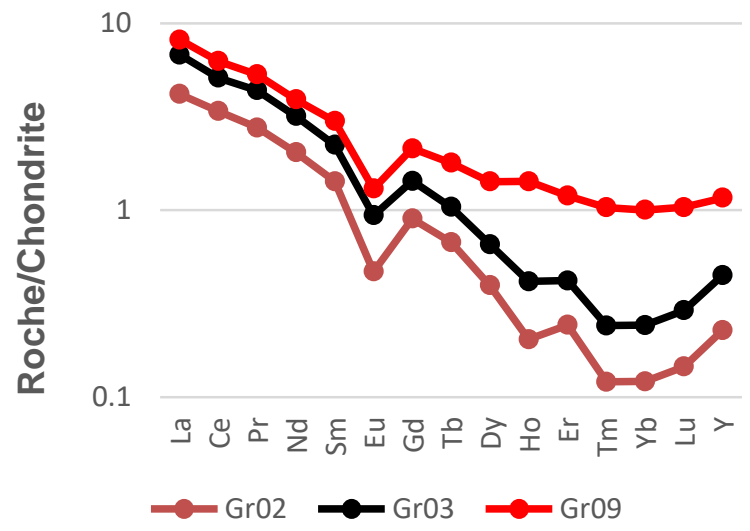

10

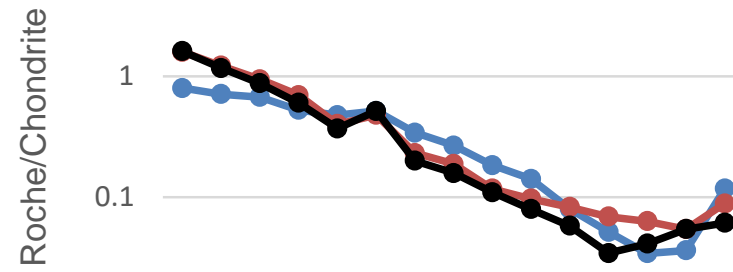

0.01

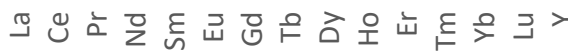

$-\mathrm{GrO1}=\mathrm{GrO4} \longrightarrow \mathrm{GrO8}$

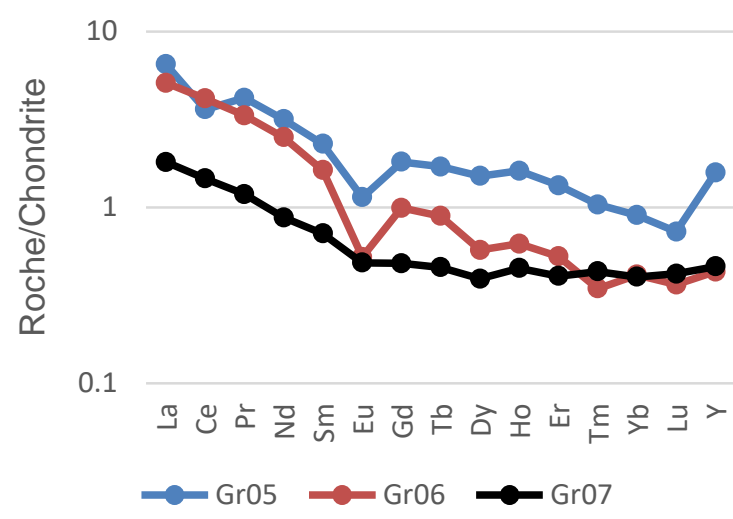

Figure 12: REE spectra normalized to chondrites of granitic rocks of the Nimba range [27]. 


\subsection{Statistical results}

3.5.1 Results of linear correlation (Bravais-Pearson) of major elements

Table 5: Linear (Bravais-Pearson) Correlation of Major Elements

\begin{tabular}{|c|c|c|c|c|c|c|c|c|c|c|}
\hline & Al2O3 & $\mathrm{CaO}$ & $\mathrm{Fe} 2 \mathrm{O} 3 \mathrm{t}$ & K2O & $\mathrm{MgO}$ & $\mathrm{MnO}$ & $\mathrm{Na} 2 \mathrm{O}$ & P2O5 & $\mathrm{SiO} 2$ & TiO2 \\
\hline \multirow[t]{3}{*}{$\mathrm{Al} 2 \mathrm{O3}$} & & $-0,0529$ & $-0,0748$ & $-0,1050$ & $-0,2872$ & $-0,2269$ & 0,3022 & 0,1406 & 0,0945 & 0,0095 \\
\hline & & (18) & (18) & (18) & (18) & (18) & (18) & (18) & $(18)$ & (18) \\
\hline & & 0,8348 & 0,7680 & 0,6784 & 0,2478 & 0,3652 & 0,2230 & 0,5779 & 0,7091 & 0,9703 \\
\hline \multirow[t]{3}{*}{$\mathrm{CaO}$} & $-0,0529$ & & 0,9546 & $-0,9192$ & 0,8854 & 0,9500 & $-0,8289$ & 0,2373 & $-0,9802$ & 0,8463 \\
\hline & $\begin{array}{l}(18) \\
\end{array}$ & & (18) & (18) & (18) & (18) & (18) & (18) & (18) & (18) \\
\hline & 0,8348 & & 0,0000 & 0,0000 & 0,0000 & 0,0000 & 0,0000 & 0,3431 & 0,0000 & 0,0000 \\
\hline \multirow[t]{3}{*}{$\mathrm{Fe} 2 \mathrm{O} 3 \mathrm{t}$} & $-0,0748$ & 0,9546 & & $-0,9245$ & 0,8260 & 0,9457 & $-0,7873$ & 0,3387 & $-0,9724$ & 0,9442 \\
\hline & (18) & (18) & & (18) & (18) & (18) & (18) & (18) & (18) & (18) \\
\hline & 0,7680 & 0,0000 & & 0,0000 & 0,0000 & 0,0000 & 0,0001 & 0,1691 & 0,0000 & 0,0000 \\
\hline \multirow[t]{3}{*}{$\mathrm{K} 2 \mathrm{O}$} & $-0,1050$ & $-0,9192$ & $-0,9245$ & & $-0,8358$ & $-0,8995$ & 0,5989 & $-0,4509$ & 0,9419 & $-0,8599$ \\
\hline & (18) & (18) & (18) & & (18) & (18) & (18) & (18) & (18) & (18) \\
\hline & 0,6784 & 0,0000 & 0,0000 & & 0,0000 & 0,0000 & 0,0086 & 0,0604 & 0,0000 & 0,0000 \\
\hline \multirow[t]{3}{*}{$\mathrm{MgO}$} & $-0,2872$ & 0,8854 & 0,8260 & $-0,8358$ & & 0,9248 & $-0,7363$ & 0,2160 & $-0,9211$ & 0,6503 \\
\hline & (18) & (18) & (18) & $(18)$ & & (18) & $(18)$ & $(18)$ & $(18)$ & $(18)$ \\
\hline & 0,2478 & 0,0000 & 0,0000 & 0,0000 & & 0,0000 & 0,0005 & 0,3892 & 0,0000 & 0,0035 \\
\hline \multirow[t]{3}{*}{$\mathrm{MnO}$} & $-0,2269$ & 0,9500 & 0,9457 & $-0,8995$ & 0,9248 & & $-0,8184$ & 0,1860 & $-0,9657$ & 0,8261 \\
\hline & (18) & (18) & (18) & (18) & (18) & & (18) & (18) & (18) & (18) \\
\hline & 0,3652 & 0,0000 & 0,0000 & 0,0000 & 0,0000 & & 0,0000 & 0,4600 & 0,0000 & 0,0000 \\
\hline \multirow[t]{3}{*}{$\mathrm{Na} 2 \mathrm{O}$} & 0,3022 & $-0,8289$ & $-0,7873$ & 0,5989 & $-0,7363$ & $-0,8184$ & & 0,1647 & 0,7790 & $-0,6496$ \\
\hline & (18) & (18) & (18) & (18) & (18) & (18) & & (18) & (18) & (18) \\
\hline & 0,2230 & 0,0000 & 0,0001 & 0,0086 & 0,0005 & 0,0000 & & 0,5136 & 0,0001 & 0,0035 \\
\hline \multirow[t]{3}{*}{$\mathrm{P} 205$} & 0,1406 & 0,2373 & 0,3387 & $-0,4509$ & 0,2160 & 0,1860 & 0,1647 & & $-0,3391$ & 0,4695 \\
\hline & (18) & (18) & (18) & (18) & (18) & $(18)$ & $(18)$ & & $(18)$ & $(18)$ \\
\hline & 0,5779 & 0,3431 & 0,1691 & 0,0604 & 0,3892 & 0,4600 & 0,5136 & & 0,1686 & 0,0493 \\
\hline \multirow[t]{3}{*}{$\mathrm{SiO} 2$} & 0,0945 & $-0,9802$ & $-0,9724$ & 0,9419 & $-0,9211$ & $-0,9657$ & 0,7790 & $-0,3391$ & & $-0,8738$ \\
\hline & (18) & (18) & $(18)$ & $(18)$ & (18) & (18) & $(18)$ & (18) & & $(18)$ \\
\hline & 0,7091 & 0,0000 & 0,0000 & 0,0000 & 0,0000 & 0,0000 & 0,0001 & 0,1686 & & 0,0000 \\
\hline \multirow[t]{3}{*}{$\mathrm{TiO} 2$} & 0,0095 & 0,8463 & 0,9442 & $-0,8599$ & 0,6503 & 0,8261 & $-0,6496$ & 0,4695 & $-0,8738$ & \\
\hline & (18) & (18) & (18) & $(18)$ & (18) & $(18)$ & $(18)$ & $(18)$ & $(18)$ & \\
\hline & 0,9703 & 0,0000 & 0,0000 & 0,0000 & 0,0035 & 0,0000 & 0,0035 & 0,0493 & 0,0000 & \\
\hline
\end{tabular}

Correlation, (Sample Size), P-Value

3.5.2 Results of Analyzes of the Main Components of Major Elements

Scree Plot

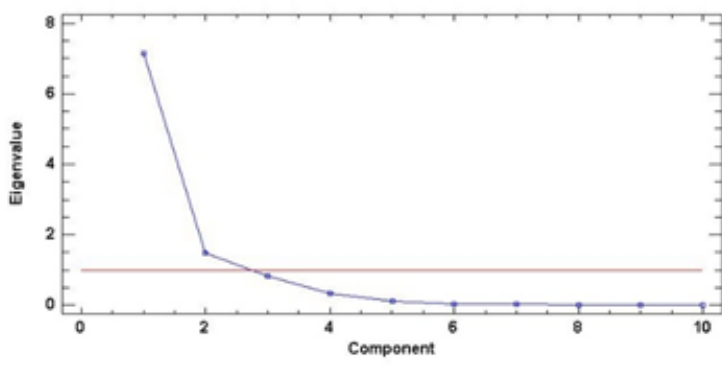

Figure 13: Diagram of the components according to their own values.

Plot of Component Weights

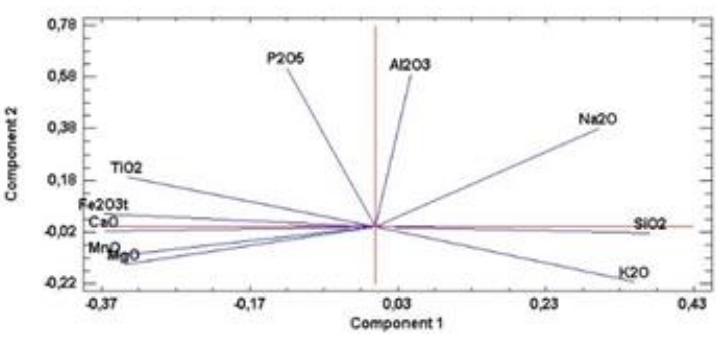

Figure 15: Diagram of the components 1 and 2 according to the weight of the major elements.

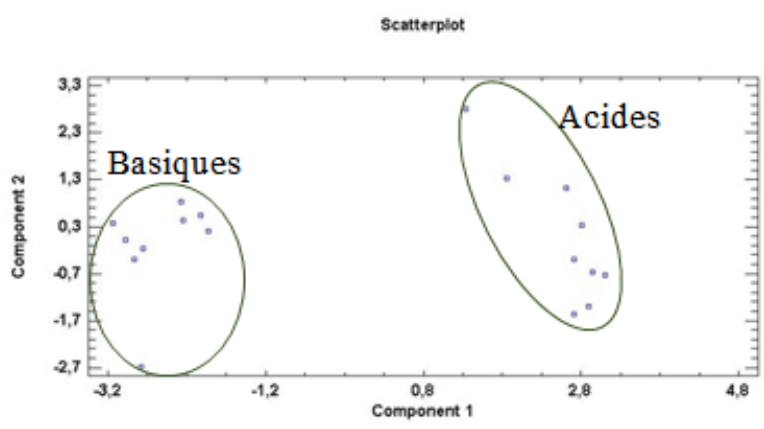

Figure 14: Diagram of the components 1 and 2 according to the samples.

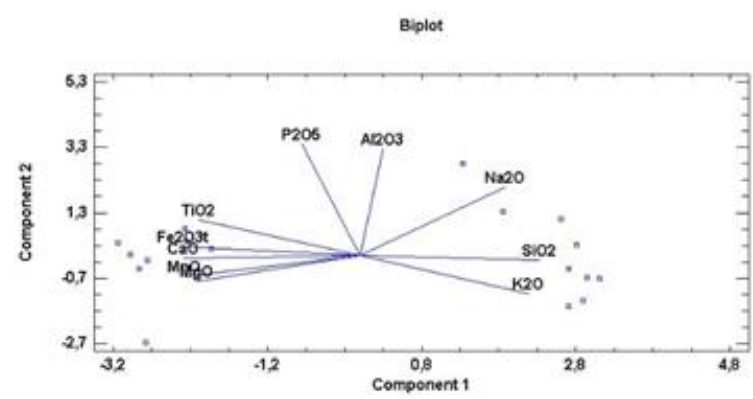

Figure 16: Diagram of the components 1 and 2 of the distribution of the rocks according to the major elements 


\subsubsection{Results of the linear correlation (Bravais-Pearson) of the trace elements}

Tables 6: Linear correlation (Bravais-Pearson) of the trace elements

\begin{tabular}{|c|c|c|c|c|c|c|c|c|c|c|c|c|c|c|}
\hline & $\mathrm{Ce}$ & Dy & $\mathrm{Er}$ & $\mathrm{Eu}$ & Gd & $\mathrm{Ho}$ & $\mathrm{La}$ & Lu & $\mathrm{Nd}$ & $\mathrm{Pr}$ & $\mathrm{Sm}$ & $\mathrm{Tb}$ & $\mathrm{Tm}$ & $\mathrm{Yb}$ \\
\hline \multirow[t]{3}{*}{$\mathrm{Ce}$} & & 0,2870 & 0,1163 & 0,4585 & 0,7205 & 0,0891 & 0,9748 & $-0,2592$ & 0,9650 & 0,9738 & 0,9042 & 0,5619 & $-0,1035$ & $-0,1404$ \\
\hline & & (18) & (18) & (18) & (18) & (18) & (18) & (18) & (18) & (18) & (18) & (18) & (18) & (18) \\
\hline & & 0,2482 & 0,6457 & 0,0556 & 0,0007 & 0,7253 & 0,0000 & 0,2989 & 0,0000 & 0,0000 & 0,0000 & 0,0152 & 0,6827 & 0,5784 \\
\hline \multirow[t]{3}{*}{ Dy } & 0,2870 & & 0,9733 & 0,7575 & 0,8535 & 0,9698 & 0,4034 & 0,7575 & 0,4872 & 0,4435 & 0,6474 & 0,9505 & 0,8845 & 0,8411 \\
\hline & (18) & & (18) & (18) & (18) & (18) & (18) & (18) & (18) & (18) & (18) & (18) & (18) & (18) \\
\hline & 0,2482 & & 0,0000 & 0,0003 & 0,0000 & 0,0000 & 0,0969 & 0,0003 & 0,0403 & 0,0653 & 0,0037 & 0,0000 & 0,0000 & 0,0000 \\
\hline \multirow[t]{3}{*}{ Er } & 0,1163 & 0,9733 & & 0,6476 & 0,7314 & 0,9951 & 0,2232 & 0,8770 & 0,3137 & 0,2658 & 0,4892 & 0,8718 & 0,9612 & 0,9337 \\
\hline & (18) & (18) & & (18) & (18) & (18) & (18) & (18) & (18) & (18) & (18) & (18) & (18) & (18) \\
\hline & 0,6457 & 0,0000 & & 0,0037 & 0,0006 & 0,0000 & 0,3733 & 0,0000 & 0,2049 & 0,2864 & 0,0394 & 0,0000 & 0,0000 & 0,0000 \\
\hline \multirow[t]{3}{*}{$\mathrm{Eu}$} & 0,4585 & 0,7575 & 0,6476 & & 0,8066 & 0,6405 & 0,6001 & 0,3013 & 0,6470 & 0,6192 & 0,7217 & 0,8017 & 0,5064 & 0,4116 \\
\hline & (18) & (18) & (18) & & (18) & (18) & (18) & (18) & (18) & (18) & (18) & (18) & (18) & (18) \\
\hline & 0,0556 & 0,0003 & 0,0037 & & 0,0001 & 0,0042 & 0,0085 & 0,2244 & 0,0037 & 0,0061 & 0,0007 & 0,0001 & 0,0320 & 0,0896 \\
\hline \multirow[t]{3}{*}{$G d$} & 0,7205 & 0,8535 & 0,7314 & 0,8066 & & 0,7117 & 0,8011 & 0,3585 & 0,8591 & 0,8320 & 0,9438 & 0,9683 & 0,5430 & 0,4795 \\
\hline & (18) & (18) & (18) & (18) & & (18) & (18) & (18) & (18) & (18) & (18) & (18) & (18) & (18) \\
\hline & 0,0007 & 0,0000 & 0,0006 & 0,0001 & & 0,0009 & 0,0001 & 0,1441 & 0,0000 & 0,0000 & 0,0000 & 0,0000 & 0,0199 & 0,0440 \\
\hline \multirow[t]{3}{*}{ Ho } & 0,0891 & 0,9698 & 0,9951 & 0,6405 & 0,7117 & & 0,1991 & 0,8760 & 0,2876 & 0,2402 & 0,4645 & 0,8590 & 0,9610 & 0,9346 \\
\hline & (18) & (18) & (18) & (18) & (18) & & (18) & (18) & (18) & (18) & (18) & (18) & (18) & (18) \\
\hline & 0,7253 & 0,0000 & 0,0000 & 0,0042 & 0,0009 & & 0,4283 & 0,0000 & 0,2471 & 0,3369 & 0,0521 & 0,0000 & 0,0000 & 0,0000 \\
\hline \multirow[t]{3}{*}{ La } & 0,9748 & 0,4034 & 0,2232 & 0,6001 & 0,8011 & 0,1991 & & $-0,2022$ & 0,9909 & 0,9969 & 0,9445 & 0,6534 & $-0,0116$ & $-0,0662$ \\
\hline & (18) & (18) & (18) & (18) & (18) & (18) & & (18) & (18) & (18) & (18) & (18) & (18) & (18) \\
\hline & 0,0000 & 0,0969 & 0,3733 & 0,0085 & 0,0001 & 0,4283 & & 0,4211 & 0,0000 & 0,0000 & 0,0000 & 0,0033 & 0,9637 & 0,7942 \\
\hline \multirow[t]{3}{*}{ Lu } & $-0,2592$ & 0,7575 & 0,8770 & 0,3013 & 0,3585 & 0,8760 & $-0,2022$ & & $-0,1080$ & $-0,1571$ & 0,0848 & 0,5632 & 0,9592 & 0,9807 \\
\hline & (18) & (18) & (18) & (18) & (18) & (18) & (18) & & (18) & (18) & (18) & (18) & (18) & (18) \\
\hline & 0,2989 & 0,0003 & 0,0000 & 0,2244 & 0,1441 & 0,0000 & 0,4211 & & 0,6697 & 0,5337 & 0,7379 & 0,0149 & 0,0000 & 0,0000 \\
\hline \multirow[t]{3}{*}{$\mathrm{Nd}$} & 0,9650 & 0,4872 & 0,3137 & 0,6470 & 0,8591 & 0,2876 & 0,9909 & $-0,1080$ & & 0,9981 & 0,9766 & 0,7263 & 0,0805 & 0,0236 \\
\hline & (18) & (18) & (18) & (18) & (18) & (18) & (18) & (18) & & (18) & (18) & (18) & (18) & (18) \\
\hline & 0,0000 & 0,0403 & 0,2049 & 0,0037 & 0,0000 & 0,2471 & 0,0000 & 0,6697 & & 0,0000 & 0,0000 & 0,0006 & 0,7507 & 0,9258 \\
\hline \multirow[t]{3}{*}{$\mathrm{Pr}$} & 0,9738 & 0,4435 & 0,2658 & 0,6192 & 0,8320 & 0,2402 & 0,9969 & $-0,1571$ & 0,9981 & & 0,9640 & 0,6902 & 0,0317 & $-0,0241$ \\
\hline & (18) & (18) & (18) & (18) & (18) & (18) & (18) & (18) & (18) & & (18) & (18) & (18) & (18) \\
\hline & 0,0000 & 0,0653 & 0,2864 & 0,0061 & 0,0000 & 0,3369 & 0,0000 & 0,5337 & 0,0000 & & 0,0000 & 0,0015 & 0,9007 & 0,9243 \\
\hline \multirow[t]{3}{*}{ Sm } & 0,9042 & 0,6474 & 0,4892 & 0,7217 & 0,9438 & 0,4645 & 0,9445 & 0,0848 & 0,9766 & 0,9640 & & 0,8465 & 0,2715 & 0,2114 \\
\hline & (18) & (18) & (18) & (18) & (18) & (18) & (18) & (18) & (18) & (18) & & (18) & (18) & (18) \\
\hline & 0,0000 & 0,0037 & 0,0394 & 0,0007 & 0,0000 & 0,0521 & 0,0000 & 0,7379 & 0,0000 & 0,0000 & & 0,0000 & 0,2758 & 0,3997 \\
\hline \multirow[t]{3}{*}{$\mathrm{Tb}$} & 0,5619 & 0,9505 & 0,8718 & 0,8017 & 0,9683 & 0,8590 & 0,6534 & 0,5632 & 0,7263 & 0,6902 & 0,8465 & & 0,7217 & 0,6749 \\
\hline & (18) & (18) & (18) & (18) & (18) & (18) & (18) & (18) & (18) & (18) & (18) & & (18) & (18) \\
\hline & 0,0152 & 0,0000 & 0,0000 & 0,0001 & 0,0000 & 0,0000 & 0,0033 & 0,0149 & 0,0006 & 0,0015 & 0,0000 & & 0,0007 & 0,0021 \\
\hline \multirow[t]{3}{*}{$\mathrm{Tm}$} & $-0,1035$ & 0,8845 & 0,9612 & 0,5064 & 0,5430 & 0,9610 & $-0,0116$ & 0,9592 & 0,0805 & 0,0317 & 0,2715 & 0,7217 & & 0,9753 \\
\hline & (18) & (18) & (18) & (18) & (18) & (18) & (18) & (18) & (18) & (18) & (18) & (18) & & (18) \\
\hline & 0,6827 & 0,0000 & 0,0000 & 0,0320 & 0,0199 & 0,0000 & 0,9637 & 0,0000 & 0,7507 & 0,9007 & 0,2758 & 0,0007 & & 0,0000 \\
\hline \multirow[t]{3}{*}{$\mathrm{Yb}$} & $-0,1404$ & 0,8411 & 0,9337 & 0,4116 & 0,4795 & 0,9346 & $-0,0662$ & 0,9807 & 0,0236 & $-0,0241$ & 0,2114 & 0,6749 & 0,9753 & \\
\hline & (18) & (18) & (18) & (18) & (18) & (18) & (18) & (18) & (18) & (18) & (18) & (18) & (18) & \\
\hline & 0,5784 & 0,0000 & 0,0000 & 0,0896 & 0,0440 & 0,0000 & 0,7942 & 0,0000 & 0,9258 & 0,9243 & 0,3997 & 0,0021 & 0,0000 & \\
\hline
\end{tabular}

Correlation, (Sample Size), P-Value

Scree Plot

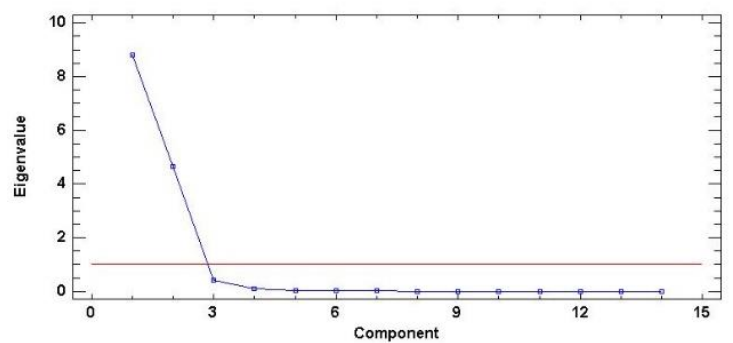

Figure 17: Diagram of the components according to the own values of the trace elements.

During the field study [photo 1] [11], two cross sections were made in the Nimba region. An ESE-WNW transversal one, in the South near the border between Guinea and Liberia. By approaching the region in its ESE part, the layers have a NW strike and a dip of about $70^{\circ}$, and in its WNW part, the layers have a dip of $80^{\circ}$ and a SE strike similar to that of the oriental set. The two parts meet at the Yâ River which is considered as a fault [figure 2]. A second transversal cross-section in the North corresponds to the range of fans. In the eastern part of the section, the layers are dipping $70^{\circ}$ to the west, while in the western part the layers have very steep dips to the east. The two parts meet at the level of the river Zié [figure 3]. The result of petrographic analyzes of rocks in the Nimba region [photo 2] reveals the presence of various minerals. Quartz and alkali are more abundant than

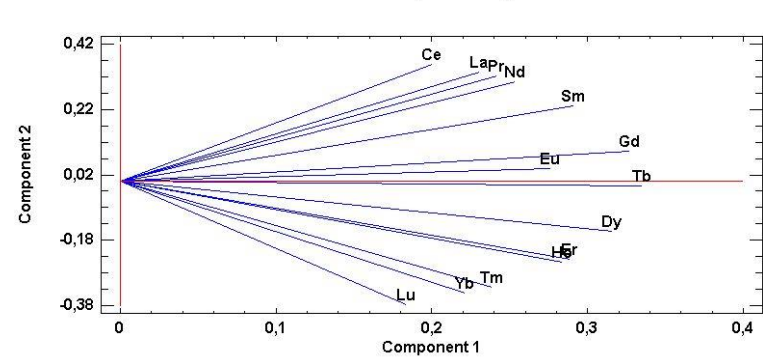

Figure 18: Diagram of the components 1 and 2 according to the weight of the trace elements.

ferromagnesian minerals [Photos $2 b, 2 c, 2 e, 2 f, 2 h, 2 i, 2 k$, $2 \mathrm{l}, 2 \mathrm{q}, 2 \mathrm{r}, 2 \mathrm{t}$ and $2 \mathrm{u}$ ] in gneisses, granites and quartzites [photos 2a , 2d, 2g, 2j, 2p and 2s]. In amphibolites and muscovitites [Figures $2 \mathrm{~m}, 2 \mathrm{v}$ and $2 \mathrm{y}$ ], ferromagnesian minerals are more abundant than quartz and alkali [Photos 2n, 2o, 2w, 2x, 2z and 2za]. In addition, the quartzitic rocks [District of Nion] [photo 2p] are characterized by the presence of zoned zircon [photo $2 q]$, open muscovite crystals in the form of " $\mathrm{S}$ " slits, quartz crystals and alkali feldspars form the "C", which gives the "C / S" structures related to the formation of "Shears Zones" [Photo 2r]. The amphibolitic rocks [District of Nion] reveal two zones, a zone of breccias and a zone of faults [photos $2 w$ and $2 x$ ]. The zone of breccias constitutes the S1 phase which is filled by the grunerite. As for the fault zone, it is filled by the microcrystals of 
quartz, magnetite and the feldspars constitute the S2 phase characterized by the displacement of blocks due to the activity of the faults.

The itabirites (name of Brazilian origin) [photos 2ab and 2ae] are metamorphosed and laminated iron oxide formations in which, on the one hand, the bands of chert or jasper were recrystallized in granular quartz and on the other hand iron is present as hematite, magnetite, and / or martite [19], [20], and [21] [photos 2ac, 2ad, 2af and 2ag] [Table 1].

The analysis of the different spectroscopies [FIG. 4] and diffractograms [FIG. 5] of the rocks confirms the result of the petrographic analysis, but also reveals the appearance of new minerals (dolomite, rutile, phlogopite, fersilicite, calcite, augite and pyrite) [Table 2].

The results of geochemical analyzes of amphibolitic and granitic rocks are summarized in Tables 3 and 4 .

The distribution of different amphibolitic and granitic rocks in the $\mathrm{Na} 2 \mathrm{O}+\mathrm{K} 2 \mathrm{O} / \mathrm{SiO} 2$ diagram of TAS [22] are located respectively in the basalts and granites fields with subalkaline tendency [figure 6]; in the AFM ternary diagram [23], these rocks are distributed in the tholeiitic and calc-alkaline fields [Figure 7].

Diagram V versus Ti / 1000 [24] shows that amphibolites fall into the MORB domain [Figure 8].

The projection of the different granite facies in the diagram A versus $B$ [25] is located in the 'Peralumineux' field [figure 9]. This character is confirmed by the high values of the molecular parameter ISA (alumina saturation index $=\mathrm{Al} 2 \mathrm{O} 3 / \mathrm{Na} 2 \mathrm{O}+\mathrm{K} 2 \mathrm{O}+\mathrm{CaO}$ ) which varies from 1.40 to 1.73 .The projection of the major elements of the different granitic rocks of the Nimba range in the Harker diagrams [figure 10], shows that $\mathrm{SiO} 2$ correlates negatively with $\mathrm{TiO} 2, \mathrm{Al} 2 \mathrm{O} 3, \mathrm{Fe} 2 \mathrm{O} 3 \mathrm{t}, \mathrm{MgO}$ and $\mathrm{CaO}$. $\mathrm{Na2O}$ has a dispersed negative correlation while K2O shows a positive correlation with $\mathrm{SiO} 2$.

This MORB trend of amphibolitic rocks is confirmed by the appearance of normalized to chondrites REE spectra [26] of N-MORBs from the depleted upper mantle. These spectra show depletion in LREE and are almost flat in HREE [Figure 11].The normalized to chondrites REE spectra (REE) of granitic rocks [27] [figure 12] allow to distinguish two types of spectra:

- the first group shows negative Eu anomalies, suggesting the fractionation of plagioclase

- the second group of spectra has positive Eu anomalies suggesting that the plagioclase has not been fractionated.

These two groups of spectra indicate that the source is rich in LREE and poor in HREE.

The linear correlation study shows that amphibolite and granite samples from the Nimba region exhibit both major elements with perfect positive linear relationships and P-Values less than 0.05 (correlations and P-values increase in the same direction) [Table 5]; and perfect negative linear relationships and P-Values less than 0.05 (correlations increase in the opposite direction of $\mathrm{P}$ Values) [Table 5].

The linear combination of the 10 variables that account for most of the data variability. Two (2) components with own values greater than or equal to 1.0 were extracted. They represent together $86.312 \%$ of the variability in original data [Figure 13].

The diagram of components 1 and 2 of the rock distribution with respect to major elements [Figure 14] shows two groups of rocks (acidic and basic).

The diagram of components 1 and 2 as a function of the weight of the major elements [figure 15] shows three (3) groups of major elements, the first formed of $\mathrm{SiO} 2$, $\mathrm{Na} 2 \mathrm{O}$ and $\mathrm{K} 2 \mathrm{O}$ correlating with each other at component 1. The second formed of $\mathrm{MgO}, \mathrm{TiO} 2, \mathrm{MnO}, \mathrm{Fe} 2 \mathrm{O} 3 \mathrm{t}$ and $\mathrm{CaO}$ correlating with each other at component 1 , but these two groups are anti-correlated with component 2 . The third group of major elements is formed of $\mathrm{Al} 2 \mathrm{O} 3$ and P2O5 which does not correlate neither with component 2 nor with the first two groups. Only $\mathrm{TiO} 2$ correlates with P2O5.

The granitic rocks of the Nimba region, rich in alkali ( $\mathrm{Na2O}$ and $\mathrm{K2O}$ ) and silica (SiO2), show magmatic differentiation. While amphibolitic rocks, rich in iron and magnesium, show no magmatic differentiation [Figure 16].The linear correlation shows that amphibolitic and granitic rocks have REE with perfect positive linear relations (correlations and $\mathrm{P}$-Values increase in the same direction) with P-Values less than 0.05 [Table 6].

The linear combinations of fourteen (14) variables that account for most of the data variability. Two (2) components with own values greater than or equal to 1.0 were extracted. Together, they represent $96.079 \%$ of the variability of the original data [Figure 17].

The diagram of components 1 and 2 according to the weight of the REE [figure 18] shows two (2) groups. The first is formed of $\mathrm{Ce}, \mathrm{La}, \mathrm{Pr}, \mathrm{Nd}, \mathrm{Sm}, \mathrm{Gd}$, and Eu which correlate with each other at component 1 . The second is formed of Tb, Dy, Er, Ho, Tm, Yb and Lu which correlate with each other at component 1 . The two REE groups are not anti-correlated to component 2 , this is confirmed by the hypothesis of the linear correlation method.

\section{Synthesis and Conclusion}

The Nimba region, located in the south-east of Guinea, on the border between Guinea, Liberia and Ivory Coast, is a mountainous region which together constitutes the chain of Nimba, one of the highest summits of West Africa (the Richard Molard mountain with 1752 m of altitude). The Precambrian of the Nimba region is largely correlated with that of Brazil and Venezuela. The similarity of age between some Venezuelian metadiabases and amphibolites from Liberia has been well established by [28] and [29]. The Nimba region is characterized by metavolcanic and metasedimentary rocks.

The petrographic studies of these rocks show that the non-ferritic quartzitic rocks in the southern part of the region [Nion District] are characterized by the presence of zoned zircon. The result of these studies shows that zircons have been dated to $2600 \mathrm{Ma}$ [30] which corresponds to the age of the eburnian orogeny. In addition, ' $C$ / S' shear phenomena have been observed, as in the case of schistosed granites (with C / S structure) of deformed zones (for example, the Oulmes granitic complex in Morocco) [31]. Then, zones of breccias and faults were observed in the amphibolitic rocks [Nion District]. Normal contact with the underlying rocks is only 
visible south of Nion [32]. In other parts of the region, however, amphibolite does not contain zones of breccias or of faults, but it does reveal the arrangement of minerals. These amphibolites are of the grunerite type (cummingtonite family) containing iron (magnetite, pyrite); they are often associated with large deposits of iron.

Geochemical analyzes of rocks in the Nimba region reveal two types of magmatism: i) acidic magmatism with $\mathrm{SiO} 2$ content between $66.64 \%$ and $74.89 \%$ (granitic rocks), which was emplaced by a magmatic differentiation manifested by the reduction of ferromagnesian mineral contents, ferro-titanic oxides and those of plagioclase and by an increase in the alkali feldspar content on the one hand; ii) basic magmatism with $\mathrm{SiO} 2$ content between $46.7 \%$ and $49.68 \%$ (amphibolitic rocks) with no magmatic differentiation, hence the use of $\mathrm{V}$ versus $\mathrm{Ti} / 1000$ [26] ] which makes it possible to locate these rocks in the field of MORB, corresponding to the depletion of the upper mantle and a setting up of the region by rifting.

Linear correlation studies of major elements of rock exhibit both positive and negative linear relationships. In the case of REE, the correlation shows a perfect positive linear relationship. The analysis of major element components (PCA) confirms the magmatic differentiation of granitic rocks (Figure 16). On the other hand, the amphibolitic rocks do not show any magmatic differentiation (figure 16). This is due to the weathering and climatic conditions of the region (the level of rainfall and dense forest that maintain a low temperature).

The Nimba region contains significant iron resources estimated to more than 300 million tonnes at $60-70 \%$ iron [33], which have been known for a long time and whose extensions into Liberia had been the subject of a mining. These are BIF (Banded Iron Formations) formations made of alternating quartzites / ironiferous quartzites, which are known in many Archaean and Proterozoic cratons and give high-amount and large-tonnage mineralizations.

The ferruginous quartzites at the origin of the deposits are attributed to the precipitation of a silico-ferruginous gel in a closed basin, practically protected from other terrigenous inputs [34]. This massive precipitation of dissolved iron results from an $\mathrm{O} 2$ enrichment of the Archaean and Paleoproterozoic atmosphere. Ore formation (hematite \pm goethite \pm magnetite) is believed to be essentially supergenic [34].

\section{References}

[1]. M. Bonhomme, (1962), Contribution à l'étude géochronologique de la plate-forme de l'Ouest Africain, Thèse, Ann, Faculté des Sciences, Université Clermont Ferrand, GeolMineral, 5, 60p.

[2]. M. Boher, (1991), Croissance crustale en Afrique de l'Ouest à $2.1 \mathrm{Ga}$. Apport de la géochimie isotopique, These, Université Nancy, 180p.

[3]. A. Potrel, J. J. Peucat, C. M. Fanning, B. Auvray, P. J. Burg C.Caruba, (1996) 3,5 Ga old terrains in the West Africa Craton, Mauritania. Journal of the Geological Society, London, Vol. 153, pp. $507-510$

[4]. V. I. Mamedov, Y.V. Bouffev, Y.A. Nikitine, (2010), Géologie de la République de Guinée, Géoprosoects Ltd, Moscou, Volume $1,166 \mathrm{p}$.

[5]. A. Obermuller, (1941), Description pétrographique et étude géologique de la région forestière de la Guinée Francaise, Thèse Université d'Alger, Bull. №5, Serv, Min. A. O. F.
[6]. M. Lamotte, G Rougerie, (1952), Coexistence de trois types de modelé dans les chaînes quartzitiques du Nimba et Simandou (Haute-Guinée Française), Ann. de Géogr., 61, Paris, pp.432-342. [7] J.W. Berger, (1774), Geology, Geochemestry, and Origin of the Nimba Itabirite and Associated Rocks, Nimba Country, Liberia, Economic Geology, 69, pp.80-92.

[8].J. W. Berge, K. Johansson, J. Jack, (1977), Geology and origin of the hematite ores of the Nimba range, Liberia, Economic Geology, 72, pp.582-607

[9]. A. Misra, P. Raucq, (1984), Itabirites et minerais de fer des Monts Nimba (Guinée), Ann., Soc. Geol, Belg,107, pp. 285-301.

[10]. D. Bering, J. Brinckmann, N. Camara, M. Diawara, L. Gast, S.Kéita, M. Giroux, D. Patrick, J. J. Lefèbvre, L. Wright, (1998) Evaluation de l'inventaire des Ressources Minérales de Guinée. Conakry, CPDM, $109 \mathrm{p}$.

[11]. Google earth, Image 2017 CNES / Digital Globe, Données cartographiques. Image satellitaire de la région de Nimba disponible sur le lien

https://www.google.fr/maps/@7.4331161,-

$8.3838118,32749 a, 35 y, 36.16 \mathrm{t} /$ data $=! 3 \mathrm{~m} 1 ! 1 \mathrm{e} 3$. Consulté le $06 / 062017$.

[12]. rruff, Search RRUFF Sample Data, Le spectroscopie de référence de l'anorthite disponible sur le lien : http://rruff.info/anorthite/display=default/R040059. Consulté le $17 / 06 / 2017$.

[13]. rruff, Search RRUFF Sample Data, Le spectroscopie de référence $\mathrm{du}$ quartz disponible sur le lien http://rruff.info/anorthite/display=default/R100170 Consulté le $17 / 06 / 2017$

[14]. rruff, Search RRUFF Sample Data, Le spectroscopie de référence du quartz disponible sur le lien : http://rruff.info/quartz/display=default/R040031. Consulté le 18/06/2017.

[15]. rruff, Search RRUFF Sample Data, Le spectroscopie de référence de la muscovite disponible sur le lien http://rruff.info/muscovite/display=default/R040104. Consulté le $20 / 06 / 2017$.

[16]. rruff, Search RRUFF Sample Data. Le spectroscopie de référence du grunérite disponible sur le lien http://rruff.info/grunerite/display=default/R040150. Consulté le 20/06/2017

[17]. rruff, Search RRUFF Sample Data. Le spectroscopie de référence de la magnétite disponible sur le lien : http://rruff.info/magn\%C3\%A9tite/display=default/R060222. Consulté le 20/06/2017.

[18]. MinerologyDatabase, X-Ray Powder Diffraction. Les données des différentes minéraux de la Diffrraction des Rayons $X$ sont disponibles sur le lien :http://webmineral.com/MySQL/xray.php\#.Wele4jvMQ1k.

Consulté le 15/06/2017.

[19]. J. Dorr, N. Van, A. L. M. Barbosa, (1963), Geology and ore deposits of the itabira district, Minas Gerais, Brazil; U. S Geological Survey Professional Paper 341c, 110p.

[20]. J. B. Pemorene, (1964), Geology and ore deposits of the Belo Horizone, Itabirite and Macacos Quadrangles, Minas Gerais, Brazil, U.S. Geological Survey Professional Paper 341-D, $84 p$.

[21]. R. M. Wallace, (1965), Geology and Mineral resources of the Pico de Itabirito district, Minas Gerias, Brazil, U. S. Geological Survey Professional Paper 341F, 68p.

[22]. R. W. Le Maitre, A. Streckeisen, B.Zanettin, M. J. Le Bas, B. Bonin, P. Bateman, (2002), Igneous rocks: A classification and glossary of terms: Recommendations of the International Union of Geological Sciences Subcommission on the Systematics of Igneous Rocks; Cambridge University Press, $252 \mathrm{p}$

[23]. T. N. Ivrine, W. R. A. Baragar, (1971), A guide to the chemical classification of the common volcanic rocks, Canadian Jour, Earth Sciences, 8, pp523-548. 
[24]. J. W. Shervais, (1982), Ti/V plotd and the petrogenesis of modern and ophiolitic lavas, Earth and Planetary Sciences Letters, 59, pp.101-118.

[25]. F. Debon, P. Le Fort, (1983), A chemical-mineralogical classification of common plutonic rocks and associations, Trans, Roy, Soc, Edinburgh Earth Sci, 73, pp.135-149.

[26]. L, A, Haskin, T. R, Wildeman, M. A. Haskin, (1968), An accurate procedure for the determination of the rare earths by neutron activation, Journal of Radioanalytical Chemistry, 1,pp.337-348.

[27]. N. Nakamura, (1974), Determination of REE, Ba, Fe, Mg $\mathrm{Na}$, and $\mathrm{K}$ in carbonaceous and ordinary chondrites, Geochimica et Cosmochimica Acta 38, pp.757-775.

[28]. T. C. Onstott, R. B. Hargraves, (1981), Proterozoic transcurrent tectonics : Paleomagnetic evidence from Venezuella and Africa, Nature, 289, pp. 131-13 7.

[29]. T. C. Onstott, J. Dorbor, (1981), 48Ar/49Ar and Paleomagnetic results from Liberia and tectonic evolution of the Precambrian APW database of the West African Shield, J. Afr. EarthSci, 6, pp. 537-552.
[30]. GUERROT, C. (1998): -Résultats d'études sur les monts Nimba, note BRGM, SMN,ANA/ISO98/232, 17p.

[31]. O. S. Ait, (1986), Modalités de mise en place d'un pluton granitique et ses relations avec la déformation régionale : l'exemple du granite hercynien d'Oulmès (Maroc central), Thèse Univ, Rennes, $224 \mathrm{p}$.

[32]. D. Thieblemont, J. C. Goujou, C. Delor, A. Cocherie, J. M Lafon, M Tegyey, H Theveniaut, A. Lacomme, M. Bah, A Balde, H. Sane, H. SALL, S. Souaré, J. Touré, (1999), BRGM, DNRGH, Notice explicative de la carte géologique de la Guinée à $1 / 200$ 000, Feuille $N^{\circ} 34-33$ N'Zérékoré-Tinsou. -Guinée (GIN): Ministère des Mines, de la Géologie et de I'Environnement, 31

[33]. V. I. Mamedov, Y.V. Bouffev, Y.A. Nikitine, (2010), Banque de données sur les gisements et indices des minéraux utiles, Volume 2, Géoprospects Ltd, Conakry-Moscou., 266.p.

[34]. A. Misra, P. Raucq, (1984), Itabirite et minerai de fer des Monts Nimba (Guinée), Bull Séanc, Acad. R. Sci, Outre-Mer, 30, pp.285-301. 